\title{
Variations in rotation rate and polar motion of a non-hydrostatic Titan
}

\author{
Alexis Coyette $^{\mathrm{a}, \mathrm{b}, *}$, Rose-Marie Baland ${ }^{\mathrm{b}}$, Tim Van Hoolst ${ }^{\mathrm{b}, \mathrm{c}}$ \\ ${ }^{a}$ Earth and Life Institute, UCL, Place Louis Pasteur 3, Louvain-la-Neuve B-1348, Belgium \\ ${ }^{\mathrm{b}}$ Royal Observatory of Belgium, Ringlaan 3, Brussels B-1180, Belgium \\ ${ }^{c}$ Instituut voor Sterrenkunde, KU Leuven, Celestijnenlaan 200D, Leuven B-3001, Belgium
}

\section{A R T I C L E I N F O}

\section{Article history:}

Received 26 October 2017

Revised 29 January 2018

Accepted 2 February 2018

Available online 8 February 2018

\section{Keywords:}

Titan

Libration

Interior structure

\begin{abstract}
A B S T R A C T
Observation of the rotation of synchronously rotating satellites can help to probe their interior. Previous studies mostly assume that these large icy satellites are in hydrostatic equilibrium, although several measurements indicate that they deviate from such a state. Here we investigate the effect of non-hydrostatic equilibrium and of flow in the subsurface ocean on the rotation of Titan. We consider the variations in rotation rate and the polar motion due to (1) the gravitational force exerted by Saturn at orbital period and (2) exchanges of angular momentum between the seasonally varying atmosphere and the solid surface. The deviation of the mass distribution from hydrostaticity can significantly increase the diurnal libration and decrease the amplitude of the seasonal libration. The effect of the non-hydrostatic mass distribution is less important for polar motion, which is more sensitive to flow in the subsurface ocean. By including a large spectrum of atmospheric perturbations, the smaller than synchronous rotation rate measured by Cassini in the 2004-2009 period (Meriggiola et al., 2016) could be explained by the atmospheric forcing. If our interpretation is correct, we predict a larger than synchronous rotation rate in the 2009-2014 period.
\end{abstract}

(c) 2018 Elsevier Inc. All rights reserved.

\section{Introduction}

Changes in the spin of solar system bodies provide insight into their deep interior, and have, for example, recently been used to determine that the core of Mercury is at least partially liquid (Margot et al., 2007) and has a radius of about $2000 \mathrm{~km}$ (Margot et al., 2012; Hauck et al., 2013; Rivoldini and Van Hoolst, 2013). In a similar approach, the librations at orbital period of Enceladus, detected on the basis of Cassini optical data, show that Enceladus has a global ocean below an about $20 \mathrm{~km}$ thick crust (Thomas et al., 2016; Čadek et al., 2016; Van Hoolst et al., 2016), a conclusion that is also compatible with an examination of gravity and shape data (Beuthe et al., 2016). Rotation variations could also be used to probe the interior of large icy satellites, in particular of Titan for which the rotation, gravity and shape have been measured by the Cassini mission.

The observational determination of rotation variations is based on measurements of the shift in orientation in inertial space of Cassini radar images taken during different flybys. Cassini radar images taken between 2004 and 2009 have shown that the rotation period of Saturn's moon Titan differs slightly from its or-

\footnotetext{
* Corresponding author at: Earth and Life Institute, UCL, Place Louis Pasteur 3, Louvain-la-Neuve B-1348, Belgium.

E-mail address: alexis.coyette@uclouvain.be (A. Coyette).
}

bital period, although firm conclusions have been difficult to obtain (Lorenz, 2008; Stiles et al., 2008; 2010). The deviation from a synchronous rotation of the ice shell is $-0.024^{\circ} \pm 0.018^{\circ} /$ year (one- $\sigma$ uncertainty, Meriggiola et al., 2016). Further analysis of the Cassini data, including data from the flybys performed since 2009, may improve the estimations of the rotational variations.

Titan is assumed to be in a mean state of rotation called a Cassini state (see e.g. Peale, 1969). It implies that Titan is in synchronous rotation and that the rate of precession of its rotation axis is close to that of the normal to its orbit. As a result, the spin axis, the normal to the orbit and the normal to the Laplace plane or inertial plane are nearly coplanar and the obliquity $\eta$ (the angle between the rotation axis and the normal to the orbital plane) is nearly constant.

Variations in the rotation rate of Titan around this mean synchronous rotation can occur for several reasons (Tokano and Neubauer, 2005; Van Hoolst et al., 2013; Richard et al., 2014). First, Titan's rotation changes with a period equal to Titan's orbital period as a result of the gravitational torque exerted by Saturn. Current theoretical models (Van Hoolst et al., 2013; Richard et al., 2014) show that the amplitudes of the diurnal rotation variations are below the detection limit related to the position error of Cassini radar images of the order of one kilometer (Meriggiola et al., 2016). Second, dynamic variations in the atmosphere (and to a less extent in the hydrocarbon lakes) of 
Titan induce changes in Titan's rotation with a main period equal to half the orbital period of Saturn. Depending on the model of the dynamics of the atmosphere and on the rotation model used (Goldreich and Mitchell, 2010; Richard et al., 2014), the maximum displacement of a given surface spot at the equator with respect to its equilibrium position without variations of the length-of-day (LOD) could be up to about $1 \mathrm{~km}$. The maximal rotation rate variations associated with LOD variations predicted for a large set of interior models is about $0.013 \%$ year (Van Hoolst et al., 2013), and is compatible with the observation in the $2-\sigma$ limit. Third, free librations with periods of the order of a year might be excited by the atmosphere of Titan, and fourth, deviations from a Keplerian orbit on different timescales introduce additional variability mostly at long periods (Richard et al., 2014; Yseboodt and Van Hoolst, 2014). Moreover, Titan might not exactly occupy the $1: 1$ spin-orbit resonance so that the rotation is non-synchronous (NSR) (Greenberg and Weidenschilling, 1984), even though the observed deviation from a synchronous rotation of the ice shell is compatible with a zero-NSR in the $2-\sigma$ limit.

In addition, the gravitational torque exerted by Saturn and the atmospheric and hydrologic torques also lead to fluctuations in the orientation of the spin axis. The position of the spin axis changes in two ways: with respect to inertial space (precession and nutations, e.g. Bills and Nimmo, 2008 and Baland et al., 2011) and with respect to the solid surface (polar motion). Polar motion of Titan due to its atmosphere and hydrocarbon lakes has recently been studied by Tokano et al. (2011) and Coyette et al. (2016). By assuming Titan (including its ocean) to be in hydrostatic equilibrium, the atmosphere forces the spin axis to follow an elliptical path with a typical amplitude of about $500 \mathrm{~m}$ in the $y$-direction and $200 \mathrm{~m}$ in the $x$ direction and a main period equal to the orbital period of Saturn. These values apply to a shell thickness of about $200 \mathrm{~km}$. For thinner shells, both the amplitude and the main period of the polar motion sensitively depend on whether a forcing period is close to the period of a free wobble mode of Titan. For shells thinner than $80 \mathrm{~km}$, the amplitude of the polar motion could reach several tens of $\mathrm{km}$ or more.

In the existing models for the librations and polar motion of Titan with an internal ocean, it is assumed that Titan is in hydrostatic equilibrium. Within the observational errors, the ratio of the degree-two gravitational coefficients agrees with that expected for a hydrostatic Titan, suggesting that Titan is indeed close to a relaxed shape (Iess et al., 2012). However, this ratio is only a necessary but not a sufficient condition for a synchronous satellite to be in hydrostatic equilibrium. The observed shape of the surface, which is more flattened at the poles than expected for hydrostatic equilibrium (Zebker et al., 2009), as well as the non-zero degreethree gravity signal (Iess et al., 2012) clearly indicate some departure from hydrostatic equilibrium. Here, we will therefore relax the assumption of hydrostatic equilibrium in the rotation model by considering the flow in the subsurface ocean and the effect of the non-hydrostatic surface of Titan on the shape of the internal boundaries.

The plan of the paper is as follows. In Section 2, we use Airylike models of isostasy to calculate the shape of the interfaces between different layers of Titan based on the observations of the degree-two gravity field (Iess et al., 2012) and topography (Zebker et al., 2009). Section 3 describes the extension of the libration theory developed in Van Hoolst et al. (2013) and the polar motion theory developed in Coyette et al. (2016) to nonhydrostatic satellites with the inclusion of a Poincare flow in the subsurface ocean. As in Coyette et al. (2016), the precession of the rotation axis of the solid layers is assumed to be known and is therefore not solved jointly with the polar motion. In Section 4, numerical results are presented for the diurnal and seasonal forced librations and polar motion. We consider the sensitivity of the li- bration to various interior structure parameters such as the rigidity and viscosity of the ice shell in the discussion (Section 5). We also study in Section 5 the possible observations of librations, LOD and polar motion. Finally, concluding remarks are presented in Section 6.

\section{Non-hydrostatic internal structure of Titan}

\subsection{Differentiation and density profile}

The mean density $\left(\bar{\rho}=1882 \pm 1 \mathrm{~kg} \mathrm{~m}^{3}\right.$, from ssd.jpl.nasa.gov) and the moment of inertia (MOI) of Titan $\left(I / M R^{2}=0.3431 \pm\right.$ 0.0004 , SOL1a of Iess et al., 2012) indicate that Titan is differentiated into an ice-ocean layer, a mantle (denoted by " $\mathrm{m}$ " in the following) and a core (c) (see also Grasset et al., 2000; Sohl et al., 2003; Tobie et al., 2005; Fortes et al., 2007). From the large tidal Love number $k_{2}=0.589 \pm 0.075$ (Iess et al., 2012) and the large value of the obliquity of Titan (Bills and Nimmo, 2008; Baland et al., 2011; 2014; Noyelles and Nimmo, 2014; Boué et al., 2017) it is assumed that the ice-ocean layer is divided into a shell ( $\mathrm{s}$ ) and a subsurface global ocean (o). The moment of inertia is derived by assuming that Titan is in hydrostatic equilibrium. Radau's equation then allows determining the moment of inertia from the degree-two coefficients of the gravitational field. Since the measured gravity and topography show that Titan deviates from hydrostatic equilibrium, we consider a range of MOI values. Gao and Stevenson (2013) showed that deviations from the hydrostatic equilibrium value of $10 \%$ or even more are possible. As in Baland et al. (2014), we consider that the true moment of inertia of Titan lies between 0.30 (Ganymede-like, e.g. Sohl et al., 2003) and 0.36 (Callisto-like, e.g. Fortes, 2012). The upper limit also corresponds to the value proposed by Hemingway et al. (2013) based on an analysis of the gravity to topography admittance. This wide range has the advantage of not excluding possible although less likely density profiles and at the same time allows better exploring consequences of a non-hydrostatic moment of inertia.

We assume that all layers are homogenous in composition. Pressure induced density variations within a layer are expected to be less than a few \% for Titan's core and below $2 \%$ for Titan's ice shell. Since these variations are smaller than the uncertainty on the mean density of the layers and density variations have a small effect on the amplitudes of the forced librations (see, e.g. Dumberry et al., 2013 for the case of librations of Mercury), we assume each layer $k$ to be of uniform density $\rho_{k}$. A spherically symmetric reference model of Titan is then determined by specifying in addition the outer radii $\left(R_{k}\right)$ of the layers.

Since a reference interior structure model is uniquely specified by 8 parameters (the densities $\rho_{k}$ and radii $R_{k}$ of all four layers) and only two quantities (the surface radius $R$ and the total mass $M_{T}$ ) are known, we consider physically plausible ranges of densities and interfaces radii as in Baland et al. (2014). The method used here to construct the interior models differs from Baland et al. (2014) as we will choose the densities and radii randomly inside the ranges instead of picking equally-spaced values. This has the advantage of giving a finer exploration of the parameters space.

We consider the density of the ice shell to be between $920 \mathrm{~kg} / \mathrm{m}^{3}$, the density of pure ice Ih at ambient pressure and temperature (e.g. Sotin et al., 1998) and $1065 \mathrm{~kg} / \mathrm{m}^{3}$, corresponding to contaminated ice and/or dense clathrates (e.g. Fortes et al., 2007). Depending on the composition (ammonia-water or salted water) and pressure, the ocean density may typically vary from $950 \mathrm{~kg} / \mathrm{m}^{3}$ to $1350 \mathrm{~kg} / \mathrm{m}^{3}$ (Croft et al., 1988; Vance and Brown, 2013). The mantle can be made of high-pressure water ices possibly contaminated with rocky materials with density between $1300 \mathrm{~kg} / \mathrm{m}^{3}$ (e.g. Grasset et al., 2000) and about $2000 \mathrm{~kg} / \mathrm{m}^{3}$. The core can be made of hydrated silicates or rocks mixed with ice and/or iron 
with a density between 2500 and $4500 \mathrm{~kg} / \mathrm{m}^{3}$ (Sohl et al., 2003). We also impose the density to decrease with increasing radius and consider an ice shell thickness $h_{s}$ between $10 \mathrm{~km}$ and $200 \mathrm{~km}$ and a minimal thickness of $5 \mathrm{~km}$ for all the layers. The mean radius of Titan's surface is $2574.73 \pm 0.09 \mathrm{~km}$ (Zebker et al., 2009).

The eight interior parameters (density and radius of the four layers) are then randomly chosen inside the ranges describe here above. If an interior model is compatible with the mean density $\bar{\rho}$ and the chosen range of moment of inertia $\left(0.30<I /\left(M R^{2}\right)<0.36\right)$, we compute the tidal Love number of this model from the analytical solution for homogeneous incompressible layers (Sabadini and Vermeersen, 2004). For the standard models, we use the following rigidities: 3.3 GPa for the shell, 4.6 GPa for the mantle and $100 \mathrm{GPa}$ for the core. In Section 5, we investigate the dependence on rigidity and viscosity. If the tidal Love number of the interior model lies in the possible range deduced from the observation of the gravity field of Titan by Cassini ( $k_{2}=0.589 \pm 0.075$, Iess et al., 2012), we keep the model as a possible interior structure of Titan. If not, the model is rejected. In total, we retained 50,000 interior structure models of Titan.

\subsection{Departure from hydrostatic equilibrium}

The observed shape of the surface of Titan does not correspond to the hydrostatic shape derived from the observed gravity field. In hydrostatic equilibrium, the surface of Titan derived from the observed gravity field is expected to be a triaxial ellipsoid with radii $a=2574.97 \mathrm{~km}, b=2574.66 \mathrm{~km}$ and $c=2574.56 \mathrm{~km}$ (Iess et al., 2010). However, the observed surface shape corresponds to an ellipsoid with radii given by $a=2575.15 \pm 0.02 \mathrm{~km}$, $b=2574.78 \pm 0.06 \mathrm{~km}$ and $c=2574.47 \pm 0.06 \mathrm{~km}$ (Zebker et al., 2009). The observed shape of Titan is therefore more flattened at the poles than expected for an hydrostatic shape. In addition to the observed shape of Titan, the presence of a non-zero degree-3 in the gravity signal (Iess et al., 2010; 2012) also clearly indicates the existence of a departure from the hydrostatic equilibrium. The non-hydrostatic departure must be partially compensated in order to be consistent with the gravity field. Similarly as Nimmo and Bills (2010), Hemingway et al. (2013) and Baland et al. (2014), we use here a compensation acting through variations in the thickness of the ice shell (Airy-type model). This leads to a thinner shell at the poles, in agreement with the maximum tidal dissipation there (Tobie et al., 2005; Beuthe, 2013). A second and different way to resolve the non-hydrostatic departure would have been to consider a compensation acting through variations in density instead of variations in thickness (Pratt-type model). Choukroun and Sotin (2012) have developed such Pratt-type model by considering a $8 \%$ increase in ice shell density near the poles as a result of an accumulation of dense ethane-rich clathrates at that places due to the larger precipitation of ethane-dominated liquids. As obliquity results for Pratt-type models do no significantly differ from those obtained with Airy-type models (Baland et al., 2014), we will here only consider an Airy-type model.

We use here the observed non-hydrostatic shape of the ice shell and consider that the mantle and core have hydrostatic shapes caused by the centrifugal, tidal and gravitational potentials. The flattenings of the interior and the mantle can be obtained from the classical theorem of Bruns that states that the topography of an equipotential surface is related to the ratio of the perturbing potential and the gravitational acceleration (e.g. Moritz, 1990, see also Appendix A. in Baland et al., 2014). We therefore have, for the core $(k=c)$ and mantle $(k=m)$

$-\frac{2}{3} \alpha_{k} R_{k}=\frac{\Phi_{k}^{20}\left(R_{k}\right)}{g\left(R_{k}\right)}$ $\frac{1}{6} \beta_{k} R_{k}=\frac{\Phi_{k}^{22}\left(R_{k}\right)}{g\left(R_{k}\right)}$,

where $\alpha_{k}=\left[\left(a_{k}+b_{k}\right) / 2-c_{k}\right] /\left[\left(a_{k}+b_{k}\right) / 2\right]$ is the polar flattening and $\beta_{k}=\left(a_{k}-b_{k}\right) / a_{k}$ is the equatorial flattening of the outer surface of layer $k$ with principal axes $a_{k}, b_{k}, c_{k}$ and $\Phi_{k}^{20}\left(R_{k}\right)$ and $\Phi_{k}^{22}\left(R_{k}\right)$ are the degree-2 order-0 and degree- 2 order-2 parts of the total perturbing potential at the outer surface of the layer $k$ (with radius $R_{k}$ ) (see Appendix A for the mathematical expressions of $\Phi_{k}^{20}\left(R_{k}\right)$ and $\left.\Phi_{k}^{22}\left(R_{k}\right)\right)$.

To complete the system, we use the surface flattenings derived from the observed shape of Titan from Zebker et al. (2009)

$\alpha_{s}=19.2236 \times 10^{-5}$

$\beta_{s}=14.36381 \times 10^{-5}$,

and the observed $C_{20}$ and $C_{22}$ gravity field coefficients $\left(C_{20}=\right.$ $-33.599 \times 10^{-6}$ and $C_{22}=10.121 \times 10^{-6}$, from less et al., 2012), which can be expressed as a function of the flattenings of the different layers (see e.g. Van Hoolst et al., 2013) as

$$
\begin{aligned}
C_{20}=- & \frac{1}{M_{T} R^{2}} \frac{8 \pi}{15}\left[\rho_{s}\left(R_{s}^{5} \alpha_{s}-R_{o}^{5} \alpha_{o}\right)+\rho_{o}\left(R_{o}^{5} \alpha_{o}-R_{m}^{5} \alpha_{m}\right)\right. \\
& \left.+\rho_{m}\left(R_{m}^{5} \alpha_{m}-R_{c}^{5} \alpha_{c}\right)+\rho_{c} R_{c}^{5} \alpha_{c}\right] \\
C_{22}= & \frac{1}{4 M_{T} R^{2}} \frac{8 \pi}{15}\left[\rho_{s}\left(R_{s}^{5} \beta_{s}-R_{o}^{5} \beta_{o}\right)+\rho_{o}\left(R_{o}^{5} \beta_{o}-R_{m}^{5} \beta_{m}\right)\right. \\
& \left.+\rho_{m}\left(R_{m}^{5} \beta_{m}-R_{c}^{5} \beta_{c}\right)+\rho_{c} R_{c}^{5} \beta_{c}\right],
\end{aligned}
$$

where $M_{T}$ is the mass of Titan.

From these eight equations (Eqs. (1) and (2) for the core and the mantle and Eqs. ((3)-(6))), we obtain the flattenings of the core, mantle, ocean and shell (see Fig. 1). They can be compared with hydrostatic flattenings obtained through integration of Clairaut's equation (e.g. Van Hoolst et al., 2008). The nonhydrostatic flattenings of the core and mantle do not deviate much from the hydrostatic ones. In contrast, the non-hydrostatic flattenings of the ocean and shell strongly differ from the hydrostatic flattenings. The non-hydrostatic polar flattening $\alpha_{o}$ is negative since it compensates for the large deviation of the surface from hydrostaticity. Most non-hydrostatic interior models are also characterized by a negative equatorial flattening $\beta_{0}$. Our flattenings of the ocean differ from those of Baland et al. (2014) due to the fact that we choose to constrain the tidal Love number $k_{2}$ in the $1-\sigma$ range. Using a $2-\sigma$ range, we would have obtained the same behavior as in Baland et al. (2014) with a broader range for $\alpha_{0}$ and $\beta_{0}$ in the presence of thick ice shells. By construction, the nonhydrostatic flattenings of the shell are the same for all the interior models. For the hydrostatic models, the flattenings of the shell present a small variability related to the range in mean moment of inertia of the interior models. The strong difference between the non-hydrostatic and hydrostatic flattenings of the ocean can have a large impact on the MOI difference $\left(B_{s}-A_{s}\right)$, especially for thin shells (see Fig. 2) and therefore on the rotation of the shell.

\section{Libration and polar motion for a non-hydrostatic Titan}

\subsection{Angular momentum equations}

We study the rotation of Titan from the angular momentum equation, or Euler-Liouville equation, that expresses the basic physical principle that the time-derivative of the angular momentum $\vec{H}$ of the rotating body is equal to the total applied torque $\vec{\Gamma}$. 

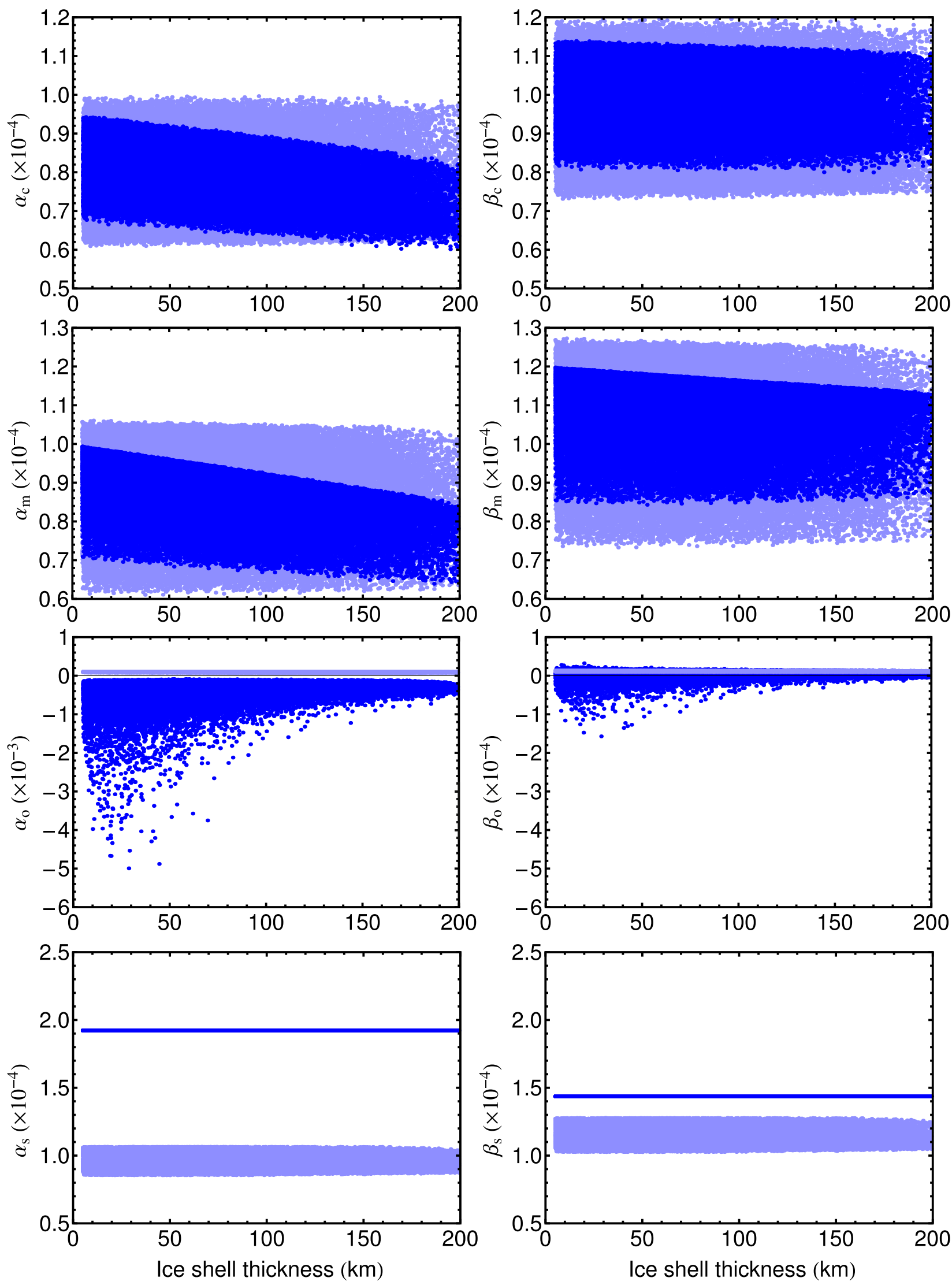

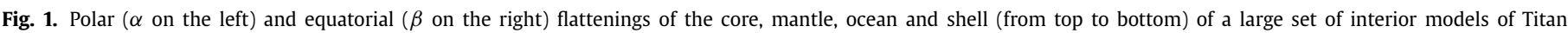

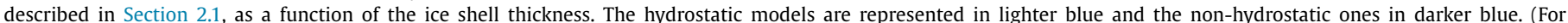
interpretation of the references to color in this figure legend, the reader is referred to the web version of this article.) 


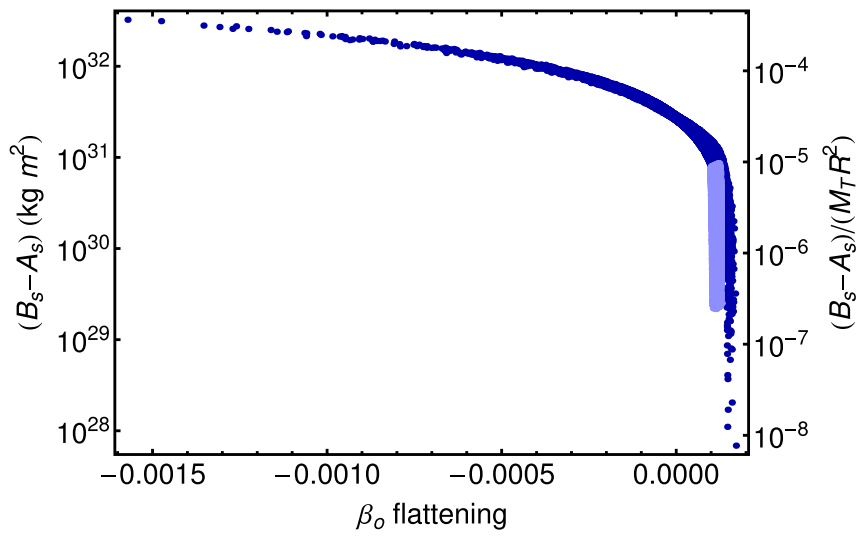

Fig. 2. MOI difference $\left(B_{s}-A_{s}\right)$ (and normalized MOI difference $\left(B_{s}-A_{s}\right) /\left(M_{T} R^{2}\right)$ at the right) as a function of the equatorial flattening of the ocean $\beta_{o}$ for the hydrostatic (lighter blue) and non-hydrostatic (darker blue) models of Titan. (For interpretation of the references to color in this figure legend, the reader is referred to the web version of this article.)

As we consider interior models of Titan composed of two solid regions separated by the ocean, we have three angular momentum equations, one for the shell (s), one for the ocean (o) and one for the interior (denoted by $i$ and comprising the core and mantle). The angular momentum equations for the shell, the ocean and the interior are (see Mathews et al., 1991)

$\frac{d \vec{H}_{s}}{d t}+\vec{\omega}_{s} \times \vec{H}_{s}=\vec{\Gamma}_{s}$

$\frac{d \vec{H}_{o}}{d t}+\vec{\omega}_{s} \times \vec{H}_{o}=\vec{\Gamma}_{o}$

$\frac{d \vec{H}_{i}}{d t}+\vec{\omega}_{i} \times \vec{H}_{i}=\vec{\Gamma}_{i}$

We choose to express the equations for the shell and for the ocean in the Body Frame (BF) of the shell and the equation for the interior in the $\mathrm{BF}$ of the interior. The $\mathrm{BF}$ of a solid layer is the frame related to the principal moments of inertia $A_{k}<B_{k}<C_{k}$ of that layer. Here $\vec{H}_{k}$ and $\vec{\omega}_{k}=\left(\omega_{k}^{x}, \omega_{k}^{y}, \omega_{k}^{z}\right)$ are the angular momentum and the rotation vector of the layer $k$, respectively. $(x, y, z)$ is the generic name given to the coordinates of the BF corresponding to the three principal axes of a solid layer (shell or interior). The angular momenta are described in Section 3.1.1.

In comparison with rotation studies of Mathews et al. (1991) for the Earth or Dumberry and Wieczorek (2016) for the Moon, we here study a system of only three equations. We do not study the relative motion of the $\mathrm{BF}$ of the interior with respect to the BF of the shell, as in Mathews et al. (1991) (see their fourth equation). We also do not study the precession and nutation of the shell rotation axis in space, which would correspond to the fifth equation introduced in Dumberry and Wieczorek (2016). As Euler's kinematic equations (e.g. Lambeck, 1980) show, polar motion is always associated with nutation, and vice-versa. However, these equations also show that long-period polar motion is associated with much smaller nutation and that long-period nutation in space leads to much smaller polar motion amplitude. Long-period polar motion is therefore almost disconnected from nutation, making it possible to study polar motion independently from nutation. In contrast, diurnal polar motion in the $\mathrm{BF}$ is coupled with nutation and is not considered here. We tested that nutation only significantly influences polar motion for periods shorter than about ten Titan days. These short-periods will therefore not be considered in the atmospheric forcing of polar motion, and also in the external gravitational forcing by Saturn. We will see that this amounts simply to neglecting the obliquity of the solid layers (see Section 3.1.1). In a similar approximation, Cassini state models can be developed independently by neglecting the polar motion (e.g. Baland et al., 2011, for an hydrostatic satellite). Librations and length-of-day variations are not affected by our choice of setting obliquities to zero.

Due to the synchronous rotation, the rotation rate of a solid layer can be written as $\omega_{k}^{z}=n+\dot{\gamma}_{k}$ where $n$ is the mean motion of Titan and $\gamma_{k}(t)$ the libration, or small deviation from the rotation angle of the layer $k$ from the rotation angle determined by the constant rotation rate $n$. The two small components $\left(\omega_{k}^{x}, \omega_{k}^{y}\right)$ describe the polar motion of that layer. The first two components of each angular momentum equation (Eqs. (7)-(9)) will be used to study the polar motion of the solid layers of Titan while the librations or length-of-day (LOD) variations will be extracted from their third component.

The total torques $\vec{\Gamma}_{k}$ on the right hand side of Eqs. ((7)-(9)) are the sum of the gravitational torques $\vec{\Gamma}_{k}^{e}$ and $\vec{\Gamma}_{k}^{g}$ exerted on layer $k$ by Saturn and by the other internal layers of Titan, respectively, on layer $k . \vec{\Gamma}_{k}$ also includes the pressure torque $\vec{\Gamma}_{k}^{p}$ exerted by the liquid ocean on the interfaces with layer $k$. This pressure torque is divided into a hydrostatic pressure torque $\vec{\Gamma}_{k}^{p, \mathrm{H}}$ and a non-hydrostatic pressure torque $\vec{\Gamma}_{k}^{p, \mathrm{NH}}$. In addition, the atmosphere (and lakes) of Titan also exerts a torque $\vec{\Gamma}^{\text {Atm }}$ on the shell. In Section 3.2, we determine expressions for all these torques.

\subsubsection{Angular momenta}

The angular momentum of any solid layer $k$ can be written, in the $\mathrm{BF}$ of that layer, as

$\vec{H}_{k}=\mathbf{I}_{k} \vec{\omega}_{k}$,

where

$\mathbf{I}_{k}=\left(\begin{array}{ccc}A_{k}+c_{k}^{11} & c_{k}^{12} & c_{k}^{13} \\ c_{k}^{12} & B_{k}+c_{k}^{22} & c_{k}^{23} \\ c_{k}^{13} & c_{k}^{23} & c_{k}+c_{k}^{33}\end{array}\right)$

is the inertia tensor of layer $k$ with principal moments $A_{k}, B_{k}$ and $C_{k}$ and increments $c_{k}^{i j}$ (see Appendix B). As all large icy satellites in synchronous rotation, Titan has a main ellipsoidal shape with moments of inertia $A<B<C$ due to rotation and static tides. The small inertia increments $c_{k}^{i j}$ represent deviations from this ellipsoidal shape of layer $k$ that arise due to rotational and tidal deformations of Titan.

To the first order in the small quantities $\omega_{k}^{x}, \omega_{k}^{y}$, $\dot{\gamma}_{k}$ and $c_{k}^{i j}$, we have

$\vec{H}_{k}=\left(\begin{array}{c}A_{k} \omega_{k}^{x}+n c_{k}^{13} \\ B_{k} \omega_{k}^{y}+n c_{k}^{23} \\ C_{k}\left(n+\dot{\gamma}_{k}\right)+n c_{k}^{33}\end{array}\right)$.

We divide the ocean into two parts: a bottom part (that we designate by the subscript $o b$ ) that is aligned with the interior and a top part (subscript ot) aligned with the shell. Since the top and bottom parts of the ocean are aligned with the shell and the interior, respectively, the principal moments of the top ocean $A_{o t}, B_{o t}$ and $C_{o t}$ are defined with respect to the same principal axes as the shell while the principal moments of the bottom ocean $A_{o b}, B_{o b}$ and $C_{o b}$ are defined with respect to the same principal axes as the interior. By using the rotation matrix $\mathbf{R}_{\mathrm{BF}}$ from the $\mathrm{BF}$ of the interior to the BF of the shell (see Eq. (C.12)), the total angular momentum of the ocean is then given, in the $\mathrm{BF}$ of the shell, by

$\left.\vec{H}_{o}\right|_{\mathrm{BF}}=\left(\mathbf{I}_{o t}+\mathbf{R}_{\mathrm{BF}} \mathbf{I}_{o b} \mathbf{R}_{\mathrm{BF}}^{-1}\right) \vec{\omega}_{o}$,

where $\vec{\omega}_{0}$ is the rotation vector of the ocean with respect to the $\mathrm{BF}$ of the shell. By taking into account the fact that Titan is in a 
Cassini state (see Eqs. (12)-(15) of Coyette et al., 2016), we then find, correct up to the first order in small quantities $\omega_{k}^{x / y}$ and obliquity $\eta_{k}$

$\left.\vec{H}_{o}\right|_{\mathrm{BF}_{s}}=\vec{H}_{o t}+\vec{H}_{o b}+C_{o b}\left(\begin{array}{c}\Delta \omega^{x}-n \Delta \eta \sin (M+\omega) \\ \Delta \omega^{y}-n \Delta \eta \cos (M+\omega) \\ 0\end{array}\right)$,

where we use the notation $\Delta \eta=\eta_{s}-\eta_{i}, \Delta \omega^{x}=\left(\omega_{s}^{x}-\omega_{i}^{x}\right)$ and $\Delta \omega^{y}=\left(\omega_{s}^{y}-\omega_{i}^{y}\right)$. Here $\vec{H}_{o t}$ and $\vec{H}_{o b}$ are the angular momentum of the top and bottom parts of the ocean expressed in the BF of the adjacent solid layer:

$\vec{H}_{o t}=\left(\begin{array}{c}A_{o t} \omega_{o}^{x}+n c_{o t}^{13} \\ B_{o t} \omega_{o}^{y}+n c_{o t}^{23} \\ C_{o t}\left(n+\dot{\gamma}_{o}\right)+n c_{o t}^{33}\end{array}\right)$

$\vec{H}_{o b}=\left(\begin{array}{c}A_{o b}\left(\omega_{o}^{x}-\Delta \omega^{x}+n \Delta \eta \sin (M+\omega)\right)+n c_{o b}^{13} \\ B_{o b}\left(\omega_{o}^{y}-\Delta \omega^{y}+n \Delta \eta \cos (M+\omega)\right)+n c_{o b}^{23} \\ C_{o b}\left(n+\dot{\gamma}_{o}\right)+n c_{o b}^{33}\end{array}\right)$.

The angular momentum of the ocean depends on the polar motion and obliquity difference between the shell and the interior. The dependence on the polar motion components is essential to our model while the obliquity terms can be neglected as we do not study here the diurnal polar motion. The diurnal polar motion has an influence smaller than $3 \%$ on the polar motion amplitude, an influence that is smaller than the uncertainties due to the layer' densities and radii. Therefore, neglecting the diurnal terms does not significantly influence our results for the polar motion of the shell.

\subsection{Torques}

\subsubsection{Pressure torque}

The pressure of the subsurface ocean acting on its top and bottom surfaces exerts a torque on the shell and on the interior given by

$\vec{\Gamma}_{S}^{p}=\int_{S_{0}}\left(\vec{r}_{o} \times \hat{n}_{o}\right) P\left(r_{o}, \varphi, \lambda\right) d S$

$\vec{\Gamma}_{i}^{p}=-\int_{S_{i}}\left(\vec{r}_{i} \times \hat{n}_{i}\right) P\left(r_{i}, \varphi, \lambda\right) d S$.

The pressure torque exerted on the ocean is given by

$\vec{\Gamma}_{o}^{p}=-\vec{\Gamma}_{s}^{p}-\vec{\Gamma}_{i}^{p}$.

In Eqs. (17) and (18), $S_{o}$ and $S_{i}$ are the outer and inner surfaces bounding the ocean and $\hat{n}_{0}$ and $\hat{n}_{i}$ are the outward unit normal on these surfaces. The top and bottom surfaces of the ocean are oblate spheroids deformed by tides and rotation. The local radius $\vec{r}_{k}$ of a layer $k$ is given by Eq. (C.3). The pressure gradient inside the subsurface ocean can be obtained from the Navier-Stokes equation expressed as

$$
\begin{aligned}
\nabla P= & -\rho_{o}\left(\nabla W+\nabla \Phi_{\mathrm{int}}+\vec{\omega}_{s / i} \times\left(\vec{\omega}_{s / i} \times \vec{r}\right)\right. \\
& \left.+\dot{\vec{\omega}}_{s / i} \times \vec{r}+2 \vec{\omega}_{s / i} \times \vec{v}_{r}+\frac{d \vec{v}_{r}}{d t}\right) .
\end{aligned}
$$

Here the first and second terms are related to the pressure field induced by $W$ and $\Phi_{\text {int }}$, the external and internal gravitational potential, respectively. The third term of Eq. (20) represents the centrifugal term due to the rotation of the reference frame corotating with the adjacent solid layer with a rotation speed $\vec{\omega}_{s / i}$ and the fourth term arises due to the time variation of this rotation speed. The fifth term represents the Coriolis effect due the relative velocity of the fluid $\vec{v}_{r}$. Finally, the last term represents the total derivative of the fluid velocity.

If the subsurface ocean is assumed to be in hydrostatic equilibrium, the right-hand side of Eq. (20) reduces to the first two components of the pressure gradient which are already included in Coyette et al. (2016). In that case, the pressure torque can be rewritten, using Gauss' theorem, as

$\vec{\Gamma}_{S}^{p, \mathrm{H}}=-\int_{V_{\text {ot }}} \vec{r} \times \rho_{0}\left(\nabla \Phi_{\text {int }}(\vec{r})+\nabla W(\vec{r})\right) d V$

$\vec{\Gamma}_{i}^{p, \mathrm{H}}=\int_{V_{i}} \vec{r} \times \rho_{0}\left(\nabla \Phi_{\text {int }}(\vec{r})+\nabla W(\vec{r})\right) d V$,

where $V_{o t}$ and $V_{i}$ are the volume of the top part of the ocean and the of the interior, respectively. The part of the pressure torque that depends on the external potential $W(\vec{r})$ will be referred to as the external hydrostatic pressure torque $\vec{\Gamma}_{s}^{p e, \mathrm{H}}$ and the part depending on the internal potential $\Phi_{\text {int }}(\vec{r})$ will be referred to as the internal hydrostatic pressure torque $\vec{\Gamma}_{s}^{p g, \mathrm{H}}$. From a comparison of expressions (21) and (22) for the pressure torques and Eqs. (27) and (34) for the gravitational torque, the hydrostatic pressure torque due to the internal gravitational potential exerted by the different layers on the shell (or interior) and to the external potential exerted by Saturn on the shell (or interior) is equal to the gravitational torque exerted on the adjacent top (or bottom) part of the ocean (see e.g. Buffett, 1996). These torques will therefore be incorporated in the gravitational torque in Sections 3.2.2 and 3.2.3.

We here also include the flow in the subsurface ocean in the form of a Poincaré flow, a uniform-vorticity flow with a residual flow required in order to have a tangential flow at the top and bottom ocean boundaries (Poincaré, 1910, see also Dehant and Mathews, 2015). This flow is the simplest flow that can be incorporated in an analytical approach and is the same as the flow in the core of the Earth used in studies of the rotation of the Earth. In the presence of such a flow, Eq. (20) can be used to obtain the following expression for the total torque acting on the ocean (see Mathews et al., 1991 and Van Hoolst and Dehant, 2002 for the triaxial case)

$\vec{\Gamma}_{o}=\vec{\omega}_{0} \times \vec{H}_{0}$.

Since the total gravitational torque (including the hydrostatic part of the pressure torque) acting on the ocean vanishes (see Van Hoolst et al., 2009 and Sections 3.2.2 and 3.2.3), the total torque is equal to the non-hydrostatic pressure torque acting on the ocean $\vec{\Gamma}_{o}^{p, \mathrm{NH}}$. Using Eq. (14) for the angular momentum of the ocean, we then find

$$
\vec{\Gamma}_{o}^{p, \mathrm{NH}}=n\left(\begin{array}{c}
\left(C_{o t}-B_{o t}\right) \omega_{o}^{y}+\left(C_{o b}-B_{o b}\right)\left(\omega_{o}^{y}-\Delta \omega^{y}\right. \\
+n \Delta \eta \cos (M+\omega))-n\left(c_{o t}^{23}+c_{o b}^{23}\right) \\
-\left(C_{o t}-A_{o t}\right) \omega_{o}^{x}-\left(C_{o b}-A_{o b}\right)\left(\omega_{o}^{x}-\Delta \omega^{x}\right. \\
+n \Delta \eta \sin (M+\omega)+n\left(c_{o t}^{13}+c_{o b}^{13}\right) \\
0
\end{array}\right) .
$$

This equation is similar to Eq. (34) of Van Hoolst (2007) (or Eq. (35) of Van Hoolst (2015) with a typo in the sign of the $\Omega^{2} c_{13}^{f}$ term of the $y$-component) where the fluid layer was assumed to extend to the center. The part of Eq. (24) that depends on the moment of inertia of the top of the ocean must be due to the shell while the part that depends on the moment of inertia of the bottom originates at interface with the interior. As 
$\vec{\Gamma}_{o}^{p, \mathrm{NH}}=-\vec{\Gamma}_{s}^{p, \mathrm{NH}}-\vec{\Gamma}_{i}^{p, \mathrm{NH}}$, we then have

$\vec{\Gamma}_{s}^{p, \mathrm{NH}}=-n\left(\begin{array}{c}\left(C_{o t}-B_{o t}\right) \omega_{o}^{y}-n c_{o t}^{23} \\ -\left(C_{o t}-A_{o t}\right) \omega_{o}^{x}+n c_{o t}^{13} \\ 0\end{array}\right)$

$$
\vec{\Gamma}_{i}^{p, \mathrm{NH}}=-n\left(\begin{array}{c}
\left(C_{o b}-B_{o b}\right)\left(\omega_{o}^{y}-\Delta \omega^{y}\right. \\
\left.+n\left(\eta_{s}-\eta_{i}\right) \cos (M+\omega)\right)-n c_{o b}^{23} \\
-\left(C_{o b}-A_{o b}\right)\left(\omega_{o}^{x}-\Delta \omega^{x}\right. \\
\left.+n\left(\eta_{s}-\eta_{i}\right) \sin (M+\omega)\right)+n c_{o b}^{13} \\
0
\end{array}\right)
$$

The z-component of Eqs. (24)-(26) is zero, indicating that the Poincare flow in the subsurface ocean only influences the polar motion and not the variations in rotation rate.

\subsubsection{External gravitational torques}

The gravitational torque exerted by Saturn on the layer $k$ is defined by

$\vec{\Gamma}_{k}^{e}=-\int_{V_{k}} \vec{r} \times \rho(\vec{r}) \nabla W(\vec{r}) d V$,

where $W(\vec{r})$ is the gravitational potential of Saturn at position $\vec{r}$ from the mass center of Titan and $V_{k}$ is the volume of layer $k$. This expression easily compares with the expression for $\vec{\Gamma}_{k}^{p e, H}$, the hydrostatic pressure torque due to the external potential (see Eqs. (21)-(22)), and shows that the torque on the ocean vanishes when only the hydrostatic part of the pressure is taken into account. The external torque on layer $k$ can also be expressed as the opposite of the torque of layer $k$ on Saturn

$\vec{\Gamma}_{k}^{e}=M_{p} \vec{r}_{p} \times \nabla W_{k}\left(\vec{r}_{p}\right)$

where $M_{p}$ is the mass of the central planet and $W_{k}\left(\vec{r}_{p}\right)$ the gravitational potential of layer $k$ at position $\vec{r}_{p}$ of the planet from the mass center of Titan. In the BF of layer $k$, and restricting $W_{k}$ expressed in terms of the components of the inertia tensor of layer $k$ (MacCullagh's theorem) to degree two, we then have (see also Baland et al., 2016)

$$
\vec{\Gamma}_{k}^{e}=3 n^{2}\left(\frac{a}{d}\right)^{3}\left(\begin{array}{c}
\left(C_{k}-B_{k}+c_{k}^{33}-c_{k}^{22}\right) r_{k}^{y} r_{k}^{z}+c_{k}^{23}\left(r_{k}^{y 2}-r_{k}^{z 2}\right) \\
+\left(c_{k}^{13} r_{k}^{y}-c_{k}^{12} r_{k}^{z}\right) r_{k}^{x} \\
\left(A_{k}-C_{k}+c_{k}^{11}-c_{k}^{33}\right) r_{k}^{x} r_{k}^{z}-c_{k}^{13}\left(r_{k}^{x 2}-r_{k}^{z 2}\right) \\
+\left(c_{k}^{12} r_{k}^{z}-c_{k}^{23} r_{k}^{x}\right) r_{k}^{y} \\
\left(B_{k}-A_{k}+c_{k}^{22}-c_{k}^{11}\right) r_{k}^{x} r_{k}^{y}+c_{k}^{12}\left(r_{k}^{x 2}-r_{k}^{y 2}\right) \\
+\left(c_{k}^{23} r_{k}^{x}-c_{k}^{13} r_{k}^{y}\right) r_{k}^{z}
\end{array}\right)
$$

where $\hat{r}_{k}=\left(r_{k}^{x}, r_{k}^{y}, r_{k}^{z}\right)$ is the unit vector pointing from the center of mass of Titan to Saturn, expressed in the BF of the layer $k . d$ is the distance from the center of mass of Saturn to the center of mass of Titan and $a$ the semi-major axis of the orbit of Titan.

In an orbital frame whose $x$-axis is chosen in the direction of Saturn, this unit vector $\hat{r}_{k}$ is expressed by construction as $(1,0,0)$. By performing transformations between this orbital frame and the $\mathrm{BF}$ of the layer $k$ and by taking into account the Cassini state, we can express the unit vector $\hat{r}_{k}$ as a function of the rotation variations (librations and polar motion), obliquity and orbit eccentricity as (see Fig. 3 and Coyette et al., 2016)

$$
\left(\begin{array}{c}
r_{k}^{x} \\
r_{k}^{y} \\
r_{k}^{z}
\end{array}\right)=\left(\begin{array}{c}
1 \\
2 e \sin M-\gamma_{k} \\
\eta_{k} \sin (M+\omega)-\frac{\omega_{k}^{x}}{n}
\end{array}\right),
$$

where $2 e \sin M$ is the equation of the center correct up to the first order in orbit eccentricity $e$. Here $\omega$ is the argument of periapsis of Titan and $M$ its mean anomaly. Eq. (30) shows that the unit vector points approximately toward the $x$-direction of the BF as expected in the Cassini state. The $y$-component of unit vector $\hat{r}_{k}$ depends on the orbit eccentricity and on the libration angle as this vector is nearly in the orbital and rotational equators. The $z$-component depends on the obliquity angle and polar motion.

The external gravitational torque (Eq. (29)) is then given, correct up to the first order in $e, \eta_{k}, \gamma_{k}, \omega_{k}^{x}$ and the incremental inertia $c_{k}^{i j}$ by

$\vec{\Gamma}_{k}^{e}=3 n^{2}\left(\begin{array}{c}0 \\ \left(A_{k}-C_{k}\right)\left(\eta_{k} \sin (M+\omega)-\frac{\omega_{k}^{x}}{n}\right)-c_{k}^{13} \\ \left(B_{k}-A_{k}\right)\left(2 e \sin M-\gamma_{k}\right)+c_{k}^{12}\end{array}\right)$.

The $x$-component of the external torque is zero correct up to the first order as the $x$-axis of the BF approximately points toward Saturn. We are then left with only torque components in the $y$ and $z$ directions that both tend to realign the long-axis of Titan in the direction of Saturn.

Including the hydrostatic pressure torque related to the external gravitational potential of Saturn (see Section 3.2.1), we find the following expression

$$
\vec{\Gamma}_{s}^{e}+\vec{\Gamma}_{s}^{p e, H}=3 n^{2}\left(\begin{array}{c}
0 \\
\left(A_{s}-C_{s}+A_{o t}-C_{o t}\right)\left(\eta_{s} \sin (M+\omega)-\frac{\omega_{s}^{\times}}{n}\right) \\
-c_{s}^{13}-c_{o t}^{13} \\
\left(B_{s}-A_{s}+B_{o t}-A_{o t}\right)\left(2 e \sin M-\gamma_{s}\right) \\
+c_{s}^{12}+c_{o t}^{12}
\end{array}\right),
$$

for the external gravitational torque acting on the shell and

$$
\vec{\Gamma}_{i}^{e}+\vec{\Gamma}_{i}^{p e, H}=3 n^{2}\left(\begin{array}{c}
0 \\
\left(A_{i}-C_{i}+A_{o b}-C_{o b}\right)\left(\eta_{i} \sin (M+\omega)-\frac{\omega_{i}^{\times}}{n}\right) \\
-c_{i}^{13}-c_{o b}^{13} \\
\left(B_{i}-A_{i}+B_{o b}-A_{o b}\right)\left(2 e \sin M-\gamma_{i}\right) \\
+c_{i}^{12}+c_{o b}^{12}
\end{array}\right),
$$

for the external gravitational torque acting on the interior. Here $\vec{\Gamma}_{s}^{p e, H}$ and $\vec{\Gamma}_{i}^{p e, H}$ represent the part of the hydrostatic pressure torque that is due to the external potential $W$ (see Eqs. (21)-(22)). The external gravitational torque (including hydrostatic external pressure torque) acting on the ocean is $\vec{\Gamma}_{o}^{e}+\vec{\Gamma}_{o}^{p e, H}=0$.

\subsubsection{Internal gravitational torques}

The presence of a subsurface ocean implies that the principal axes of the solid interior can be misaligned with those of the shell. Each layer therefore exerts a gravitational torque on the other misaligned layers. The gravitational torque acting on a layer $k$ is given by

$\vec{\Gamma}_{k}^{g}=-\int_{V_{k}} \vec{r} \times \rho_{k} \nabla \Phi(\vec{r}) d V$ 


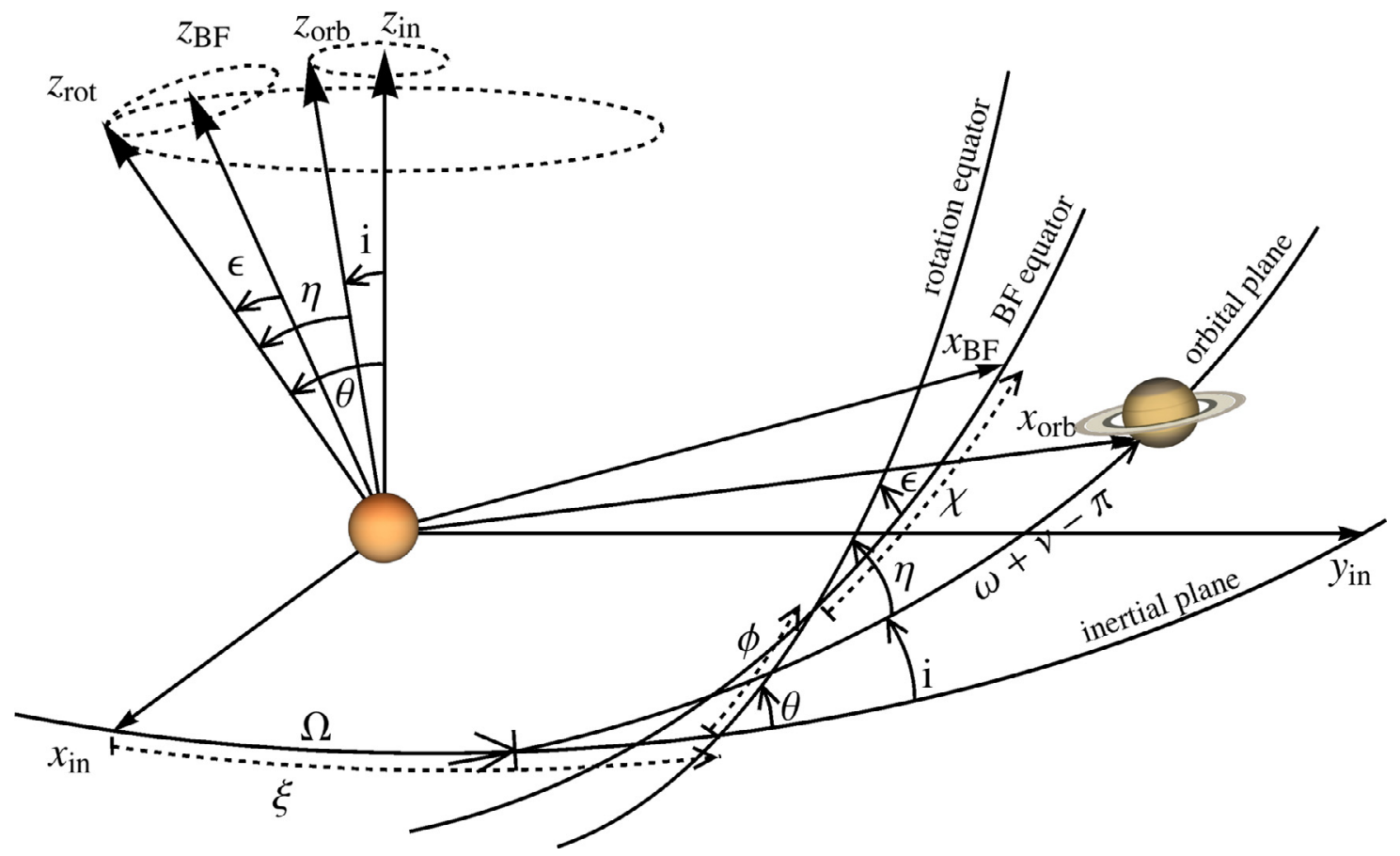

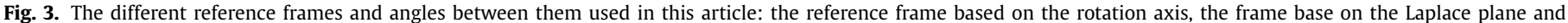
the body frame (of the shell or the interior).

where $\Phi(\vec{r})$ is the gravitational potential at position $\vec{r}$ and due to the mass distribution of the satellite misaligned with layer $k$ and $V_{k}$ is the volume of that layer.

The internal gravitational torque acting on a generic internal layer $I$ and due to the presence of a generic outer layer $O$ is described in Appendix C. The internal gravitational torques acting on a layer is due to the layers misaligned with this layer. The internal gravitational torque acting on the interior is therefore due to the top part of the ocean and the shell. Similarly, the internal gravitational torque acting on the shell is due to the interior and the bottom part of the ocean that is aligned with the interior. Using Eqs. (C.16)-(C.18) and including hydrostatic internal pressure torque, the internal gravitational torque acting on the shell is given by

$\vec{\Gamma}_{S}^{g, H}+\vec{\Gamma}_{s}^{p g, H}$

$$
\begin{gathered}
=\frac{4 \pi G}{5}\left[\left(\begin{array}{c}
\left(C_{i+o b}-B_{i+o b}\right) \rho \delta_{\alpha \beta, x}\left(\frac{\Delta \omega^{y}}{n}-\Delta \eta \cos (M+\omega)\right) \\
\left(A_{i+o b}-C_{i+o b}\right) \rho \delta_{\alpha \beta, y}\left(\frac{\Delta \omega^{x}}{n}-\Delta \eta \sin (M+\omega)\right) \\
2\left(B_{i+o b}-A_{i+o b}\right)\left[\rho_{s}\left(\beta_{o}-\beta_{s}\right)-\rho_{o} \beta_{o}\right]\left(\gamma_{s}-\gamma_{i}\right)
\end{array}\right)\right. \\
+\left(\begin{array}{c}
\left(c_{i}^{23}+c_{o b}^{23}\right) \rho \delta_{\alpha \beta, x} \\
-\left(c_{i}^{13}+c_{o b}^{13}\right) \rho \delta_{\alpha \beta, y} \\
-2\left(c_{i}^{12}+c_{o b}^{12}\right)\left[\rho_{s}\left(\beta_{s}-\beta_{o}\right)+\rho_{o} \beta_{o}\right]
\end{array}\right) \\
\left.+2\left(\begin{array}{c}
-\left(C_{i+o b}-B_{i+o b}\right)\left[\rho_{s}\left(\alpha \delta h_{s}^{\mathrm{obl}, \text { sin }}-\alpha \delta h_{o}^{\mathrm{obl}, \mathrm{sin}}\right)+\rho_{o} \alpha \delta h_{o}^{\mathrm{obl}, \mathrm{sin}}\right] \\
-\left(A_{i+o b}-C_{i+o b}\right)\left[\rho_{s}\left(\alpha \delta h_{s}^{\mathrm{obl}, \mathrm{cos}}-\alpha \delta h_{o}^{\mathrm{obl}, \mathrm{cos}}\right)+\rho_{o} \alpha \delta h_{o}^{\mathrm{obl}, \mathrm{cos}}\right] \\
\left(B_{i+o b}-A_{i+o b}\right)\left[\rho_{s}\left(\beta \delta h_{s}^{l}-\beta \delta h_{o}^{l}\right)+\rho_{o} \beta \delta h_{o}^{l}\right]
\end{array}\right)\right],
\end{gathered}
$$

where $\rho \delta_{\alpha \beta, x}=\rho_{s}\left(-2\left(\alpha_{s}-\alpha_{0}\right)+\left(\beta_{s}-\beta_{0}\right)\right)-\rho_{0}\left(2 \alpha_{o}-\beta_{0}\right)$ and $\rho \delta_{\alpha \beta, y}=\rho_{s}\left(-2\left(\alpha_{s}-\alpha_{0}\right)-\left(\beta_{s}-\beta_{0}\right)\right)-\rho_{0}\left(2 \alpha_{0}+\beta_{0}\right)$ and where we use the notation $A_{i+o b}=A_{i}+A_{o b}$ (and similarly for $B_{i+o b}$ and $\left.C_{i+o b}\right)$. The internal gravitational torque acting on the interior is given by

$\vec{\Gamma}_{i}^{g+p g, H}=-\vec{\Gamma}_{s}^{g+p g, H}$.

The internal gravitational torque (including hydrostatic internal pressure torque) acting on the ocean is $\vec{\Gamma}_{o}^{g}+\vec{\Gamma}_{o}^{p g H}=0$.
The first line of Eq. (35) expresses the gravitational torque between the static bulges of the shell and the interior. The second line of this equation is the gravitational torques between the static bulge of the shell and the periodic tidal bulge of the interior. Finally, the last line of Eq. (35) expresses the gravitational torque between the periodic tidal bulge of the shell and the static bulge of the interior.

\subsubsection{Atmospheric torque}

We use the atmospheric torque as determined by Coyette et al. (2016) based on an atmospheric Global Circulation Model (GCM) for Titan's atmosphere (Tokano, 2013). The time series of the Atmospheric Angular Momentum (AAM) cover a full Saturnian year (29.42 years) beginning at the Northern vernal equinox and are sampled 24 times per Titan day.

The three components of this atmospheric torque are expressed as time-series of the following form

$$
\begin{aligned}
\Gamma^{\mathrm{Atm}, x}= & -\omega_{s}^{y} h^{z}(0)+n h^{y}(0) \\
& +\sum_{\varpi \neq 0}\left[\Gamma_{\cos }^{\mathrm{Atm}, x}(\varpi) \cos (\varpi t)+\Gamma_{\sin }^{\mathrm{Atm}, x}(\varpi) \sin (\varpi t)\right] \\
\Gamma^{\mathrm{Atm}, y}= & \omega_{s}^{x} h^{z}(0)-n h^{x}(0) \\
& +\sum_{\varpi \neq 0}\left[\Gamma_{\cos }^{\mathrm{Atm}, y}(\varpi) \cos (\varpi t)+\Gamma_{\sin }^{\mathrm{Atm}, y}(\varpi) \sin (\varpi t)\right] \\
\Gamma^{\mathrm{Atm}, z}= & \sum_{\varpi \neq 0} \Gamma^{\mathrm{Atm}, z}(\varpi) \sin \left(\varpi t+\phi^{\mathrm{Atm}, z}(\varpi)\right) .
\end{aligned}
$$

Here

$h^{x / y / z}(0)=h_{\text {mot }}^{x / y / z}(0)+\left(1+k_{2}^{\prime}\right) h_{\text {pres }}^{x / y / z}(0)$

are the constant terms of the $x, y$ and $z$-components of the AAM and angular momentum of the lakes (see below) consisting of the effective pressure and motion terms, respectively. Due to the super-rotation of the atmosphere of Titan, $h^{z}(0)$ is the dominant term. As $h_{\mathrm{mot}}^{z}(0)$ and $h_{\mathrm{pres}}^{z}(0)$ are constant and positive while the 

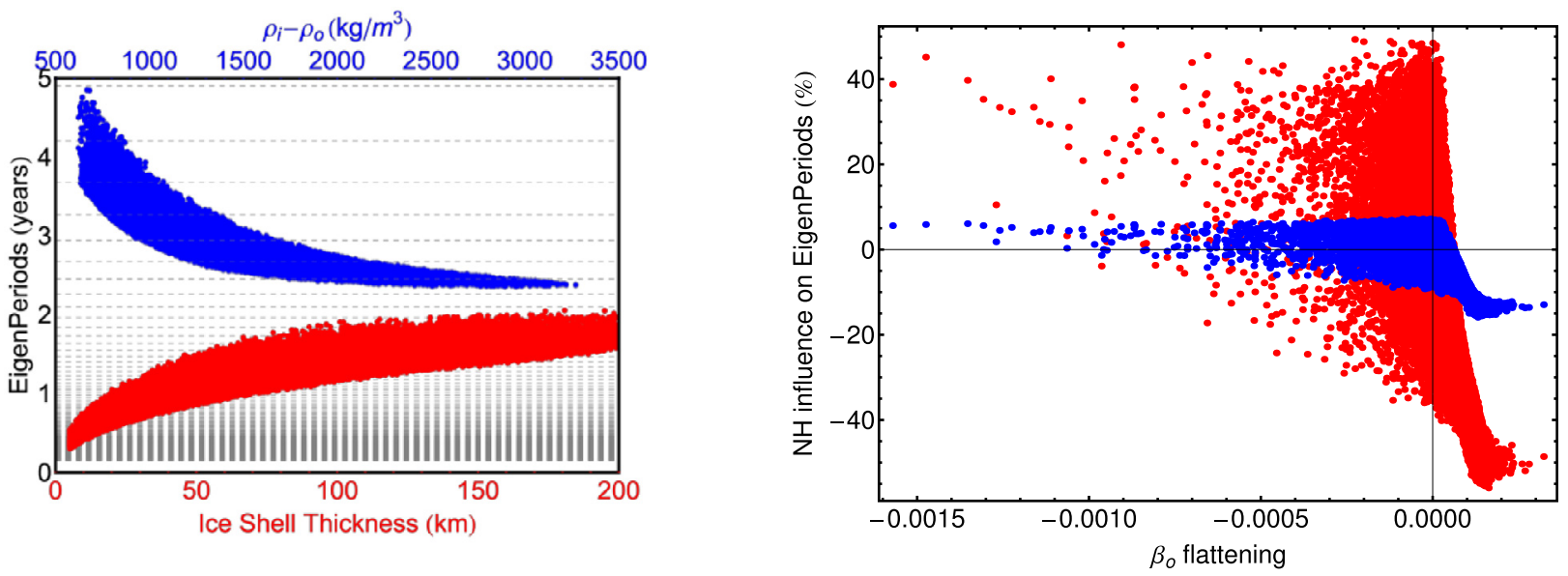

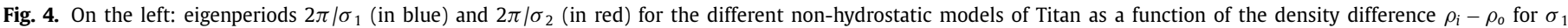

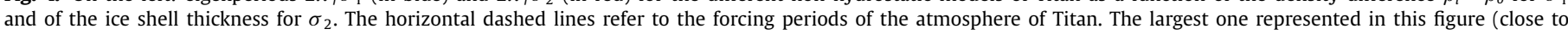

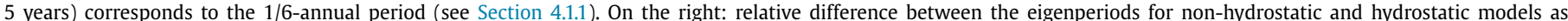

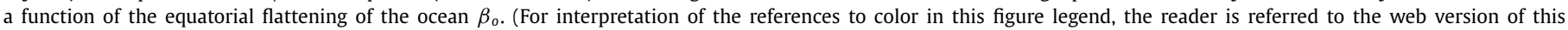
article.)

load Love number $k_{2}^{\prime}$ is negative and increases with increasing ice shell thickness, $h^{z}(0)$ increases with increasing ice shell thickness from $3.22 \times 10^{25}$ to $7.39 \times 10^{25} \mathrm{~N} \mathrm{~m} \mathrm{~s}$. As $h_{\text {pres }}^{x / y}(0)$ are negative, $h^{x}(0)$ and $h^{y}(0)$ decrease with increasing ice shell thickness, from $6.50 \times 10^{21}$ to $-1.2 \times 10^{22} \mathrm{~N} \mathrm{~m} \mathrm{~s}$ and from $-5.88 \times 10^{21}$ and $-8.71 \times 10^{22} \mathrm{~N} \mathrm{~m} \mathrm{~s}$, respectively.

Titan's hydrocarbon lakes (Tokano et al., 2014; Tokano and Lorenz, 2015) in principle also influence Titan's rotation but the time variations of the associated torque are three orders of magnitude smaller than the atmospheric torques and will be neglected here (Coyette et al., 2016). The lakes only significantly influence the constant terms of the torque acting on the surface of Titan $\Gamma^{\text {Atm, } x}(0)$ and $\Gamma^{\text {Atm, } y}(0)$ through the pressure terms of the angular momentum of the lakes. Those contributions will be included in the atmospheric torque.

\section{Results}

\subsection{Variations in rotation rate}

The equations governing the librations in longitude are the third components of the Euler-Liouville equation for the shell, the ocean and the interior (Eqs. (7)-(9)). Using Eqs. (32), (33), (35) and (36), these equations can be rewritten as (see Van Hoolst et al., 2013 for the equations for the shell and the interior and Appendix D for the definitions of the $K_{i}$ coefficients)

$C_{s} \ddot{\gamma}_{s}+K_{1} \gamma_{s}+K_{2} \gamma_{i}=4 e K_{3} \sin M+\Gamma_{\mathrm{Atm}, z}^{\mathrm{eff}}$

$C_{0} \ddot{\gamma}_{0}=e K_{0} \sin M$

$C_{i} \ddot{\gamma}_{i}+K_{4} \gamma_{s}+K_{5} \gamma_{i}=4 e K_{6} \sin M$.

The Euler-Liouville equation for the ocean (Eq. (42)) is similar to Eq. (37) of Van Hoolst (2015). Without $K_{0}$ factor that arises from the changes in the mass distribution of the ocean due to deformations ( $c_{0}^{33}$ term), the libration of the ocean disappears, leading to a constant rotation rate of the ocean. We here neglect the centrifugal part of $c_{0}^{33}$ (second term in Eq. (B.6)) that has a negligible effect on the librations and LOD (Van Hoolst et al., 2013). The mean anomaly is given by $M=M_{0}+n t$, where $M_{0}$ is the mean anomaly at Northern vernal equinox. It has a diurnal period whereas the atmospheric torque presents variations at various timescales including the diurnal period as well as shorter and larger periods. We will solve for each frequency separately.

\subsubsection{Length-of-day (LOD) variations}

For the length-of-day variations (longitudinal librations forced by the atmosphere) at atmospheric forcing periods, we drop the $\sin M$ terms in Eqs. (41)-(43). We look for solutions of the form $\gamma_{k}^{\mathrm{LOD}}(t)=g_{k}^{\mathrm{LOD}}(\varpi) \sin \left(\varpi t+\phi^{\mathrm{Atm}, z}(\varpi)\right)$ and obtain the following length-of-day amplitudes at the atmospheric frequency $\varpi$ (Eqs. (67) and (68) of Van Hoolst et al., 2013, for the solid layers)

$g_{s}^{\mathrm{LOD}}(\varpi)=\frac{\Gamma_{\mathrm{Atm}, z}(\varpi)\left(K_{5}-\varpi^{2} C_{i}\right)}{C_{i} C_{s}\left(\varpi^{2}-\sigma_{1}^{2}\right)\left(\varpi^{2}-\sigma_{2}^{2}\right)}$

$g_{0}^{\mathrm{LOD}}(\varpi)=0$

$g_{i}^{\mathrm{LOD}}(\varpi)=\frac{-\Gamma_{\mathrm{Atm}, z}(\varpi) K_{4}}{C_{i} C_{s}\left(\varpi^{2}-\sigma_{1}^{2}\right)\left(\varpi^{2}-\sigma_{2}^{2}\right)}$,

where $\sigma_{1}$ and $\sigma_{2}$ are the two frequencies of the eigenmodes of the system $((41)-(43))$

$\sigma_{1,2}^{2}=\frac{K_{1} C_{i}+K_{5} C_{s} \pm \sqrt{4\left(K_{2} K_{4}-K_{1} K_{5}\right) C_{i} C_{s}+\left(K_{1} C_{i}+K_{5} C_{s}\right)^{2}}}{2 C_{i} C_{s}}$.

The first eigenperiod (associated with $\sigma_{1}$ ) corresponds to a situation where the shell and the interior librate in phase. It ranges between 2.3 and 4.7 years (see left panel of Fig. 4). For the second eigenperiod (associated with $\sigma_{2}$ ), the shell and the interior are out of phase by $\pi$. This second period is of the order of 2 years for thick shells and decreases down to 105 days for thin shells.

The eigenperiods for non-hydrostatic models of Titan present a similar pattern as the eigenperiods for hydrostatic models as a function of ice shell thickness (see Fig. 6 of Van Hoolst et al. (2013) for $2 \pi / \sigma_{1}$ and $2 \pi / \sigma_{2}$ as a function of $h_{s}$ in the hydrostatic equilibrium case). The non-hydrostatic values for the first eigenperiod $\sigma_{1}$ are close to the hydrostatic values for this eigenperiod with a difference smaller than $20 \%$ (see right panel of Fig. 4). The effect of the deviation from hydrostaticity on the $\sigma_{2}$ eigenperiod can be larger and increases with decreasing $\beta_{0}$ flattening (see right panel of Fig. 4). 

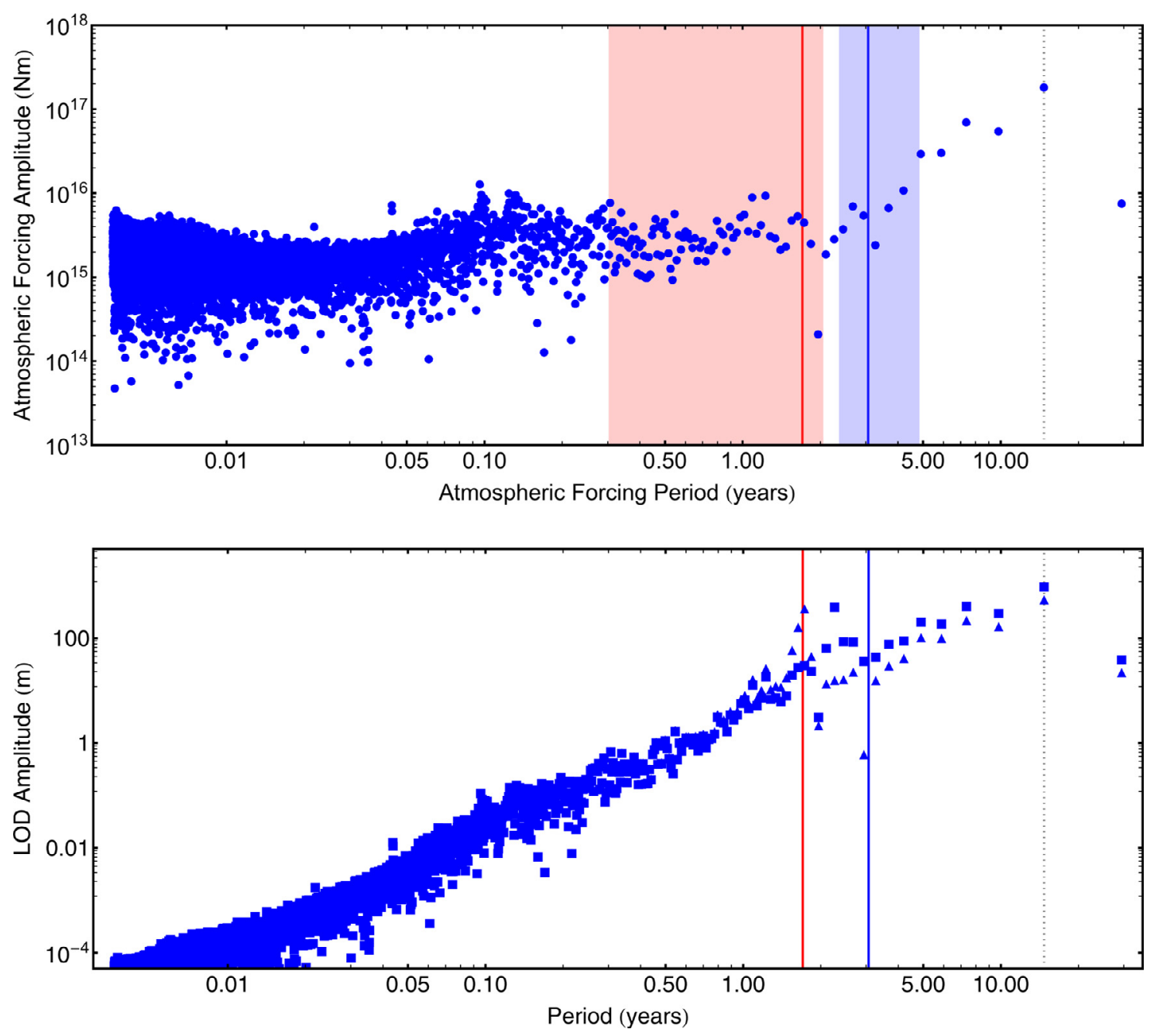

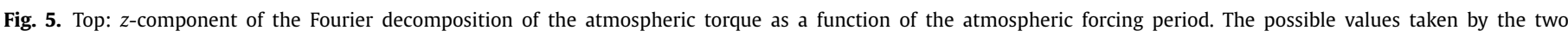

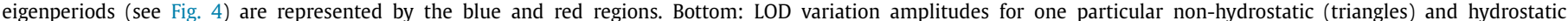

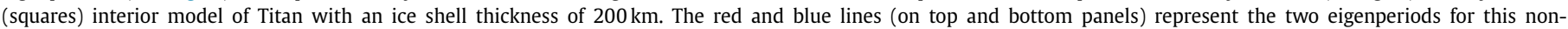

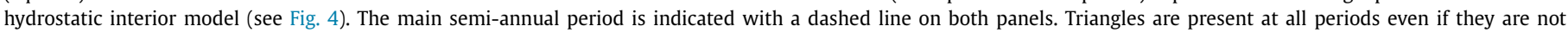

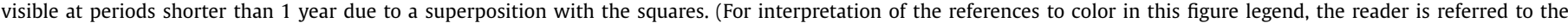
web version of this article.)

The $z$-component of the atmospheric torque (Eq. (39)) has an amplitude of $1.82 \times 10^{17} \mathrm{Nm}$ at the main semi-annual period due to seasonal variations and leads to semi-annual (period of 14.73 years) LOD variations with an amplitude of the order of $1 \mathrm{~km}$ for hydrostatic models of Titan (Van Hoolst et al., 2013). Although smaller, atmospheric forcings at shorter periods can be close enough to the eigenperiods related to the free frequencies $\sigma_{1}$ and $\sigma_{2}$ (see blue and red regions, respectively, in top panel of Fig. 5) to cause resonant amplifications of the LOD variations.

The bottom panel of Fig. 5 presents the amplitudes of the LOD variation at all atmospheric periods for one specific interior model of Titan with an ice shell thickness of $200 \mathrm{~km}$. LOD variations amplitudes are classically given in seconds but can also be expressed in meters representing the distance (at Titan's surface) between the expected position of the long axis of Titan and its actual position. For this model, the LOD amplitude is maximum at the semiannual period and decreases with decreasing period, except for atmospheric period close to the eigenperiods (red and blue lines on bottom panel of Fig. 5) where resonant amplifications of the LOD amplitudes occur.

For non-hydrostatic models of Titan, the amplitude of the LOD variations at semi-annual period is about $600 \mathrm{~m}$ for thick ice shells and ranges from $600 \mathrm{~m}$ to $2000 \mathrm{~m}$ for thin shells (see Fig. 6). The

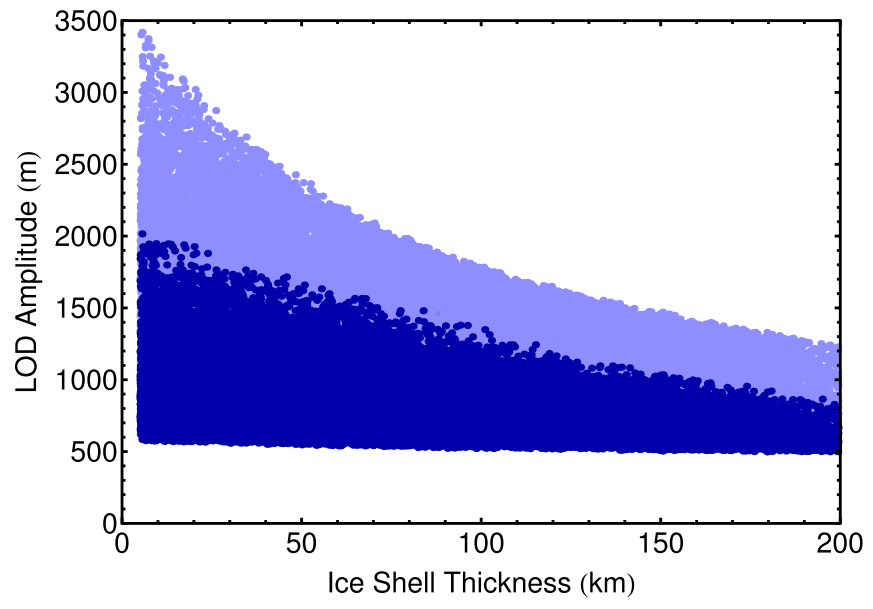

Fig. 6. Amplitude of the LOD variations at semi-annual period as a function of the ice shell thickness for hydrostatic (lighter blue) and non-hydrostatic (darker blue) models of Titan. (For interpretation of the references to color in this figure legend, the reader is referred to the web version of this article.) 
a) $1 / 10-$ annual period

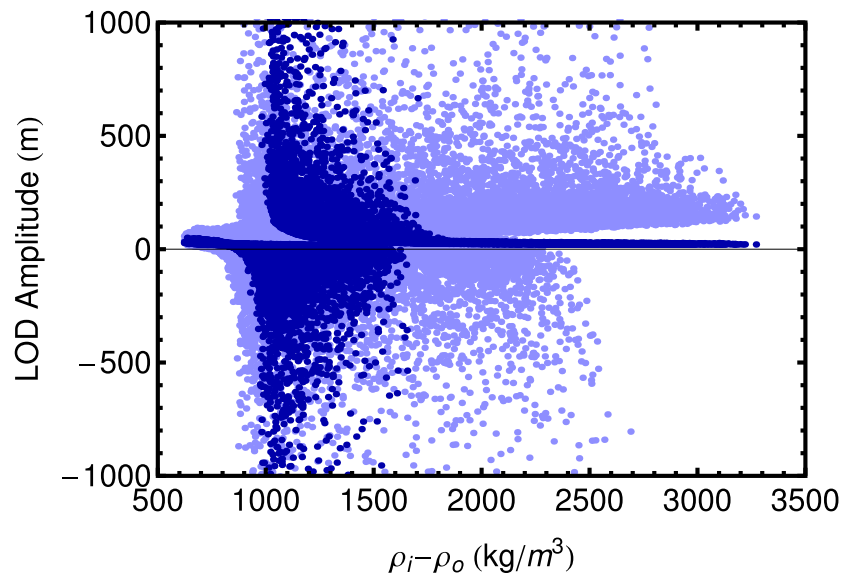

b) $1 / 30$-annual period

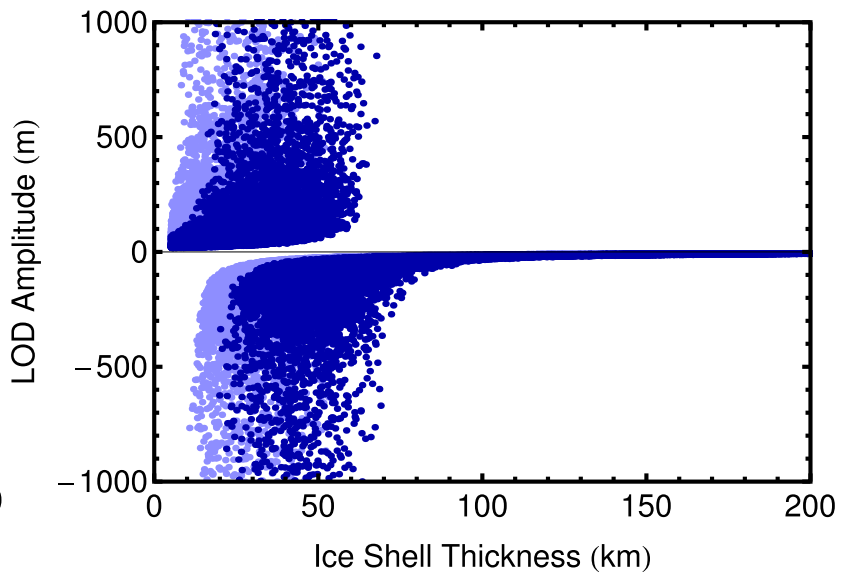

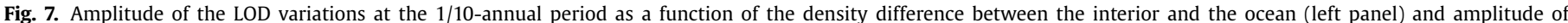

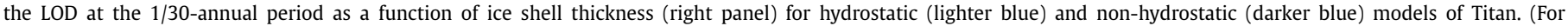
interpretation of the references to color in this figure legend, the reader is referred to the web version of this article.)

non-hydrostatic model decreases the amplitude with respect to hydrostatic models by several tens of \%. This decrease is mainly due to the increase in the coefficient $K_{1}$ as a result of the larger MOI differences $\left(B_{S}-A_{s}\right)$ and $\left(B_{o t}-A_{o t}\right)$ for the non-hydrostatic models of Titan (see Fig. 2). This coefficient divided by the polar moment of inertia of the shell can be interpreted as the squared eigenfrequency in the case of an uncoupled shell (see Eq. (41)). Analogy with the mathematical oscillator for forcing periods much larger than the eigenperiod shows that the amplitude is inversely proportional to the square of the eigenfrequency and thus to $K_{1}$ (see also Van Hoolst, 2015), explaining the smaller LOD variations due to the deviation from hydrostaticity.

The amplitude of the LOD variations at annual period (smaller than $100 \mathrm{~m}$ ), at 1/3-annual period (between $200 \mathrm{~m}$ and $600 \mathrm{~m}$ depending on the interior model), 1/4-annual period (between $200 \mathrm{~m}$ and $800 \mathrm{~m}$ depending on the interior model) and 1/5-annual period (between $100 \mathrm{~m}$ and $350 \mathrm{~m}$ depending on the interior model) are smaller than the semi-annual period amplitude as the atmospheric forcing is smaller at these periods. They present a similar increase with decreasing ice shell thickness as the semi-annual variations (not shown here).

The atmospheric forcing periods shorter than the $1 / 6$-annual period (4.91 years) can be close to the eigenperiods $2 \pi / \sigma_{1}$ or $2 \pi / \sigma_{2}$ (see Fig. 4). The amplitude of the LOD at these periods can therefore be resonantly amplified. The first eigenfrequency $\sigma_{1}$ mainly depends on the density jump between the interior and the ocean and not on the ice shell thickness (see Fig. 4). The fact to have a resonant amplification or not at a given period is therefore related to the density difference $\rho_{i}-\rho_{0}$. Resonant amplification occurs between the 1/8-annual period (3.68 years) for interior models with a density jump between the interior and the ocean of about $700 \mathrm{~kg} / \mathrm{m}^{3}$ and the $1 / 12$-annual period (2.45 years) for a density difference between the interior and the ocean larger than $2500 \mathrm{~kg} / \mathrm{m}^{3}$. Resonant amplification of the $1 / 10$-annual period occurs for interior models with a density jump of about $1100 \mathrm{~kg} / \mathrm{m}^{3}$ (see Fig. 7). For even shorter period, the amplitude of the LOD can be amplified due to a resonance with the second eigenperiod $2 \pi / \sigma_{2}$. This eigenperiod mainly depends on the ice shell thickness (see Fig. 4). For an ice shell thickness of about $150 \mathrm{~km}$, the resonant amplification will happen for a 1/18-annual period (1.64 years) while the resonance will be at a shorter period (1/30-annual period or shorter, corresponding to periods shorter than one year) for an ice shell thickness of about $50 \mathrm{~km}$ (see Fig. 7).

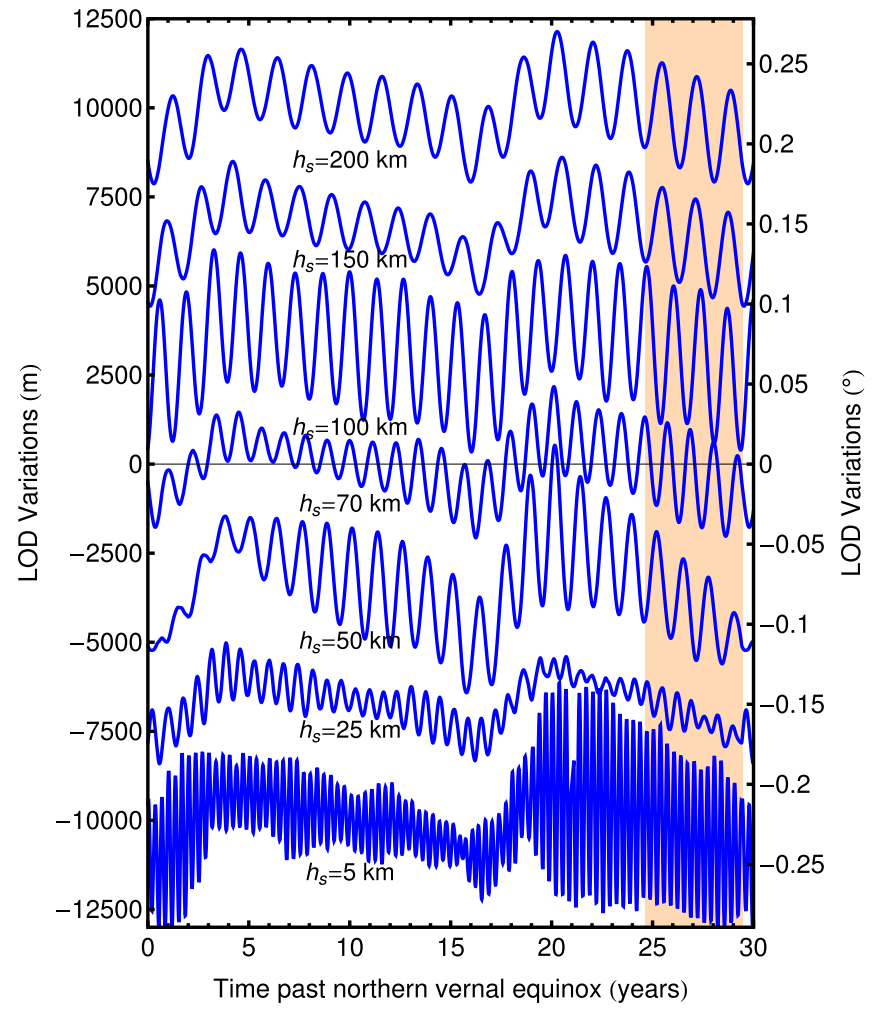

Fig. 8. LOD variations during one saturnian year for particular interior models of Titan with ice shell thickness of $200 \mathrm{~km}, 150 \mathrm{~km}, 100 \mathrm{~km}, 70 \mathrm{~km}, 50 \mathrm{~km}, 25 \mathrm{~km}$ and $5 \mathrm{~km}$ (from top to bottom). The orange region represents the period of time between June 2004 and August 2009 covering the acquisition of data that he been used in Meriggiola et al. (2016) to infer the rotational state of Titan. Each curve is shifted by $3300 \mathrm{~m}$ from the precedent curve. The LOD variations for an ice shell thickness of $70 \mathrm{~km}$ is not shifted. (For interpretation of the references to color in this figure, the reader is referred to the web version of this article.)

The LOD variations of a series of interior models of Titan with a shell density of $1000 \mathrm{~kg} / \mathrm{m}^{3}$ and various ice shell thicknesses ranging from $5 \mathrm{~km}$ to $200 \mathrm{~km}$ are presented in Fig. 8. The semi-annual LOD due to the main seasonal variation in the atmosphere of Titan is clearly apparent but superimposed on it are shorter period variations which strongly depend on the interior model. 

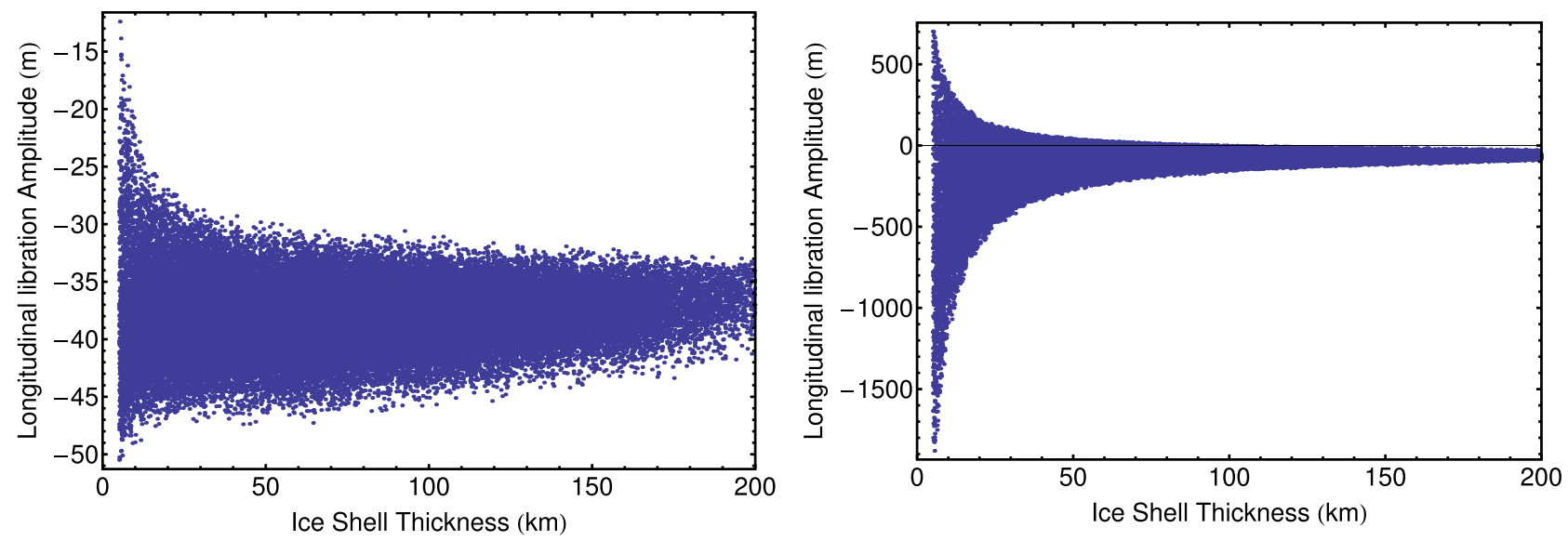

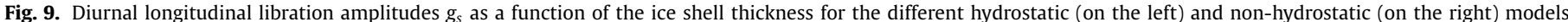
of Titan.

\subsubsection{Diurnal longitudinal librations}

For the diurnal longitudinal libration amplitude, we look for solutions of the form $\gamma_{s}(t)=g_{s} \sin M, \gamma_{0}(t)=g_{0} \sin M$ and $\gamma_{i}(t)=$ $g_{i} \sin M$. The amplitudes of the diurnal longitudinal librations for the shell, the ocean and the interior are then given by

$g_{s}=\frac{4 e\left(K_{3} K_{5}-K_{2} K_{6}-n^{2} K_{3} C_{i}\right)}{C_{i} C_{s}\left(n^{2}-\sigma_{1}^{2}\right)\left(n^{2}-\sigma_{2}^{2}\right)}$

$g_{o}=-\frac{e\left(k_{2, o b}+k_{2, o t}\right) q_{r} M_{T} R^{2}}{C_{o}}$

$g_{i}=\frac{4 e\left(K_{1} K_{6}-K_{3} K_{4}-n^{2} K_{6} C_{s}\right)}{C_{i} C_{S}\left(n^{2}-\sigma_{1}^{2}\right)\left(n^{2}-\sigma_{2}^{2}\right)}$.

The diurnal period is far away from the much longer eigenperiods. For thin shells with the shortest eigenperiods, the amplification due to the resonant amplification is smaller than $2 \%$.

For the hydrostatic interior models, the amplitude of the diurnal longitudinal libration weakly depends on the ice shell thickness (see left panel of Fig. 9), as expected due to the absence of resonant amplification (Van Hoolst et al., 2013). The amplitude of the diurnal longitudinal libration for the hydrostatic interior models is always negative (the amplitudes presented in Van Hoolst et al., 2013 are the absolute values of the amplitudes). Non-hydrostatic interior models can show a large increase of the libration amplitude up to more than a $\mathrm{km}$ for thin shells (see right panel of Fig. 9). Non-hydrostatic models with thick ice shells ( $\geq 50 \mathrm{~km}$ ) present a libration amplitude between $-270 \mathrm{~m}$ and $40 \mathrm{~m}$. This large increase of the libration amplitude is not due to a resonant amplification but rather to an increase of the moment of inertia difference $\left(B_{s}-A_{s}\right)$ and/or $\left(B_{o t}-A_{o t}\right)$ for some interior models due to the non-hydrostatic flattenings. The torque exerted by Saturn on the shell then increases (up to five times larger than for hydrostatic models), leading to larger libration amplitudes. Most longitudinal libration amplitudes are negative, but some interior models are characterized by positive libration amplitudes, meaning that the solution is in phase with the forcing. This happens for interior models with shells thinner than $100 \mathrm{~km}$ and characterised by strong negative value of $\beta_{o}$. For these models, the external coupling is smaller than the tidal torque between the static bulge of the interior and the tidal bulge of the shell, therefore leading to positive libration amplitudes.

The libration amplitude of the interior is between $15 \mathrm{~m}$ and $45 \mathrm{~m}$ and is not strongly influenced by the deviation from hydrostaticity. The libration amplitude of the ocean mainly depends on the ocean thickness and strongly increases with decreasing ocean thickness, with a libration amplitude that can reach more than $1 \mathrm{~km}$ for oceans thinner than $50 \mathrm{~km}$ (see Fig. 10).

\subsection{Polar motion}

The equations describing the polar motion are the first two components of the Euler-Liouville equation Eqs. (7)-(9). By using Eqs. (12) and (14) for the angular momentum and Eqs. (25)-(26), (32)-(33), (35)-(36) and (37)-(38) for the torques, these equations can be expressed as a system of six first-order differential equations:

$\mathbf{J}+\mathbf{K}\left(\begin{array}{c}\omega_{s}^{x}(t) \\ \omega_{s}^{y}(t) \\ \omega_{0}^{x}(t) \\ \omega_{0}^{y}(t) \\ \omega_{i}^{x}(t) \\ \omega_{i}^{y}(t)\end{array}\right)+\mathbf{N}\left(\begin{array}{c}\eta_{s} \cos (M+\omega) \\ \eta_{s} \sin (M+\omega) \\ \eta_{o} \cos (M+\omega) \\ \eta_{o} \sin (M+\omega) \\ \eta_{i} \cos (M+\omega) \\ \eta_{i} \sin (M+\omega)\end{array}\right)=\left(\begin{array}{c}\Gamma_{\mathrm{eff}}^{\mathrm{Atm}, x} \\ \Gamma_{\mathrm{eff}}^{\mathrm{Atm}, y} \\ 0 \\ 0 \\ 0 \\ 0\end{array}\right)$,

where

$\mathbf{J}=\left(\begin{array}{c}\tilde{A}_{s} \dot{\omega}_{s}^{x}(t) \\ \tilde{B}_{s} \dot{\omega}_{s}^{y}(t) \\ A_{o} \dot{\omega}_{o}^{x}(t)+\left(C_{o b}-A_{o b}\right) \Delta \dot{\omega}^{x}(t)+\frac{4}{3} q_{r} M_{T} R^{2}\left(k_{2, o b} \dot{\omega}_{i}^{x}(t)+k_{2, o t} \dot{\omega}_{s}^{x}(t)\right) \\ B_{o} \dot{\omega}_{o}^{y}(t)+\left(C_{o b}-B_{o b}\right) \Delta \dot{\omega}^{y}(t)+\frac{1}{3} q_{r} M_{T} R^{2}\left(k_{2, o b} \dot{\omega}_{i}^{y}(t)+k_{2, o t} \dot{\omega}_{s}^{y}(t)\right) \\ \tilde{A}_{i} \dot{\omega}_{i}^{x}(t) \\ \tilde{B}_{i} \dot{\omega}_{i}^{y}(t)\end{array}\right)$.

Here $\tilde{A_{k}}$ and $\tilde{B_{k}}$ are the effective equatorial moments of inertia of layer $k$

$\tilde{A}_{k}=A_{k}+\frac{4}{3} k_{2, k} q_{r} M_{T} R^{2}$

$\tilde{B}_{k}=B_{k}+\frac{1}{3} k_{2, k} q_{r} M_{T} R^{2}$

The matrices $\mathbf{K}$ and $\mathbf{N}$ are given in Appendix D. 

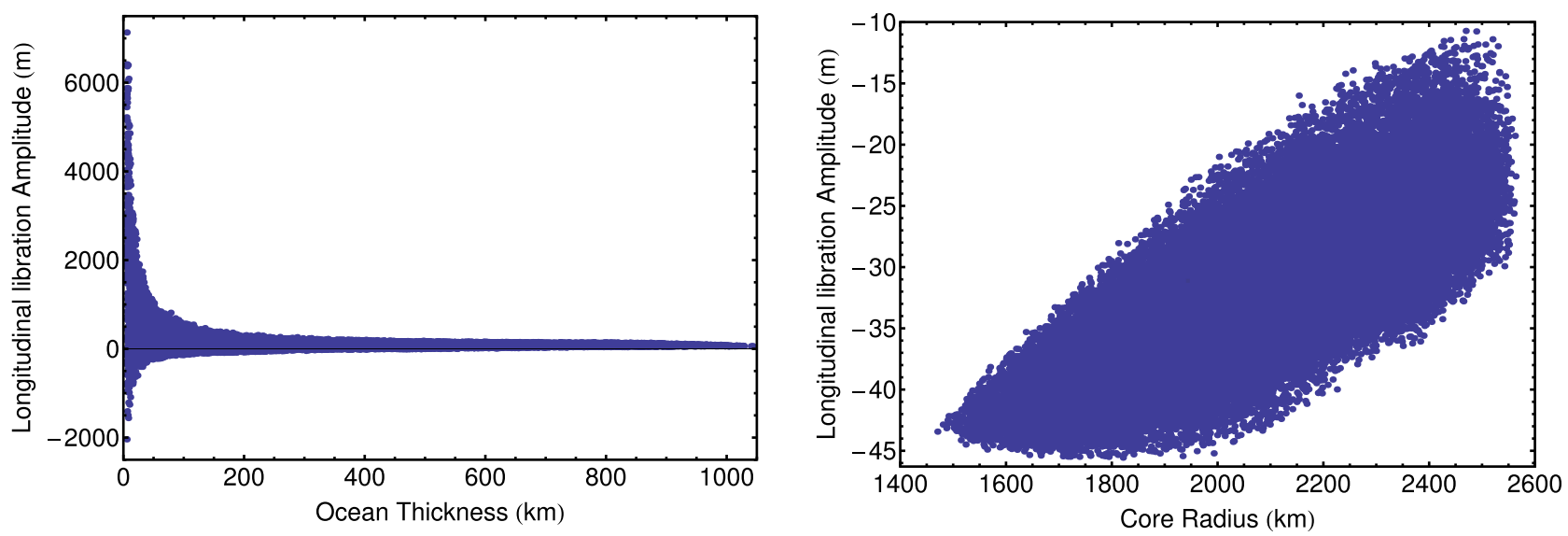

Fig. 10. Diurnal longitudinal libration amplitudes $g_{0}$ (on the left) and $g_{i}$ (on the right) as a function of the ocean thickness and of the core radius, respectively.

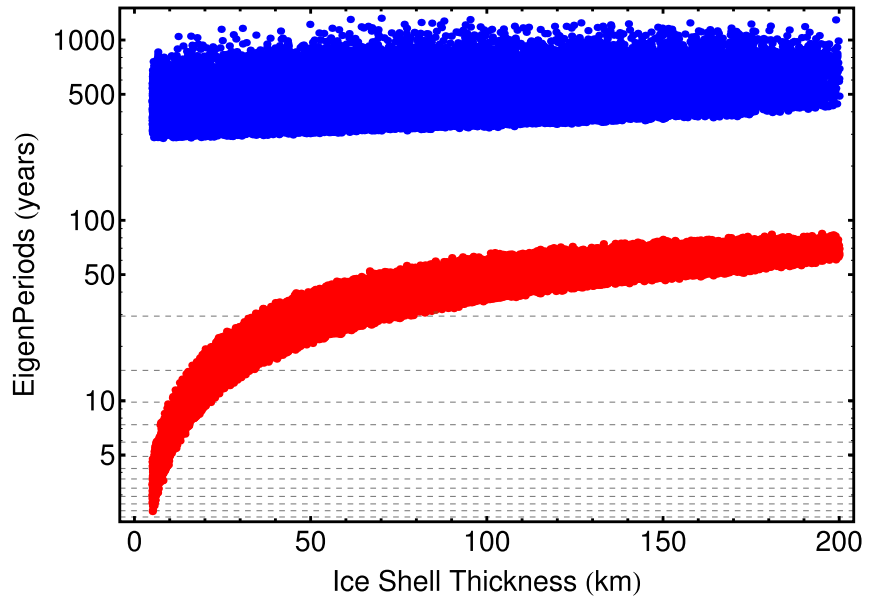

Fig. 11. Eigenperiods related to the polar motion for non-hydrostatic models of Ti$\tan$ as a function of the ice shell thickness. The CW period is represented in red while the ICW period is in blue. The dashed lines represent the different atmospheric forcing periods. (For interpretation of the references to color in this figure legend, the reader is referred to the web version of this article.)

\subsubsection{Free modes}

The free solutions of the polar motion equations are obtained by solving Eq. (51) averaged over the forcing period. The system has three free modes and although analytical expressions for their periods have been obtained, they are too long to be given here explicitly. The first free mode has a period ranging from about 300 years to more than 1000 years, much longer than the atmospheric forcing periods (see Fig. 11). By analogy with the definition used for the Earth, it corresponds to the Inner Core Wobble (ICW) of Ti$\tan$. This mode describes the prograde rotation of the figure axis of the interior about the axis of rotation of the interior while the rotation axes of the shell and of the interior remain nearly aligned with the figure axis of the shell. The second free mode has a much shorter period going from about 50 years for thick shells down to less than 2 years for very thin shells (see Fig. 11) and corresponds to the Chandler Wobble (CW) of Titan. The CW consists essentially of a prograde rigid rotation of the figure axis of the shell about the rotation axis of the shell, the rotation axes of the interior, the ocean and the shell being maintained close to each other. Finally, the third free mode is nearly diurnal in the Body Frame of Titan. It corresponds to the Nearly Diurnal Free Wobble (NDFW) of Titan that arises from a misalignement between the rotation axis of the liquid layer and the one of the shell. Since our equations are not adequate for this frequency range for polar motion because of our choice to study the polar motion independently from nutation, which is only feasible for long-period polar motion as shown by Euler's kinematic equations, we will not consider this mode any further.

The Poincare flow in the subsurface ocean lengthens the periods of the CW and of the ICW (see Fig. 12). Without a Poincaré flow, the CW eigenperiod is strongly shortened (from $50 \%$ to about $100 \%$ ). The ICW eigenperiod is shortened by $15 \%$ up to $60 \%$. The influence of the non-hydrostatic interior of Titan is smaller than $30 \%$ on the ICW and can be larger than $100 \%$ on the CW. These two effects combine in a non-linear way. As a result, in comparison with hydrostatic interior models of Titan and without a Poincaré flow (free modes presented in Coyette et al., 2016), the ICW period increases by up to $50 \%$ and the CW periods remain close to those presented in Coyette et al. (2016) for some interior models but can also be more than two times smaller for other interior models.

\subsubsection{Forced solutions}

We solve Eq. (51) for each frequency separately by writing the polar motion components as

$\omega_{s}^{x}(t)=\omega_{s}^{x, \text { off }}+\sum_{\varpi \neq 0}\left(\Omega_{s}^{x, \cos }(\varpi) \cos (\varpi t)+\Omega_{s}^{x, \sin }(\varpi) \sin (\varpi t)\right)$

$\omega_{s}^{y}(t)=\omega_{s}^{y, \text { off }}+\sum_{\varpi \neq 0}\left(\Omega_{s}^{y, \cos }(\varpi) \cos (\varpi t)+\Omega_{s}^{y, \sin }(\varpi) \sin (\varpi t)\right)$

$\omega_{o}^{x}(t)=\omega_{o}^{x, \text { off }}+\sum_{\varpi \neq 0}\left(\Omega_{o}^{x, \cos }(\varpi) \cos (\varpi t)+\Omega_{o}^{x, \sin }(\varpi) \sin (\varpi t)\right)$

$\omega_{o}^{y}(t)=\omega_{o}^{y, \text { off }}+\sum_{\varpi \neq 0}\left(\Omega_{o}^{y, \cos }(\varpi) \cos (\varpi t)+\Omega_{o}^{y, \sin }(\varpi) \sin (\varpi t)\right)$

$\omega_{i}^{x}(t)=\omega_{i}^{x, \text { off }}+\sum_{\varpi \neq 0}\left(\Omega_{i}^{x, \cos }(\varpi) \cos (\varpi t)+\Omega_{i}^{x, \sin }(\varpi) \sin (\varpi t)\right)$

$\omega_{i}^{y}(t)=\omega_{i}^{y, \text { off }}+\sum_{\varpi \neq 0}\left(\Omega_{i}^{y, \cos }(\varpi) \cos (\varpi t)+\Omega_{i}^{y, \sin }(\varpi) \sin (\varpi t)\right)$.

The first terms of these expressions are the constant solutions of Eq. (51), which correspond to an offset of the center of the polar 

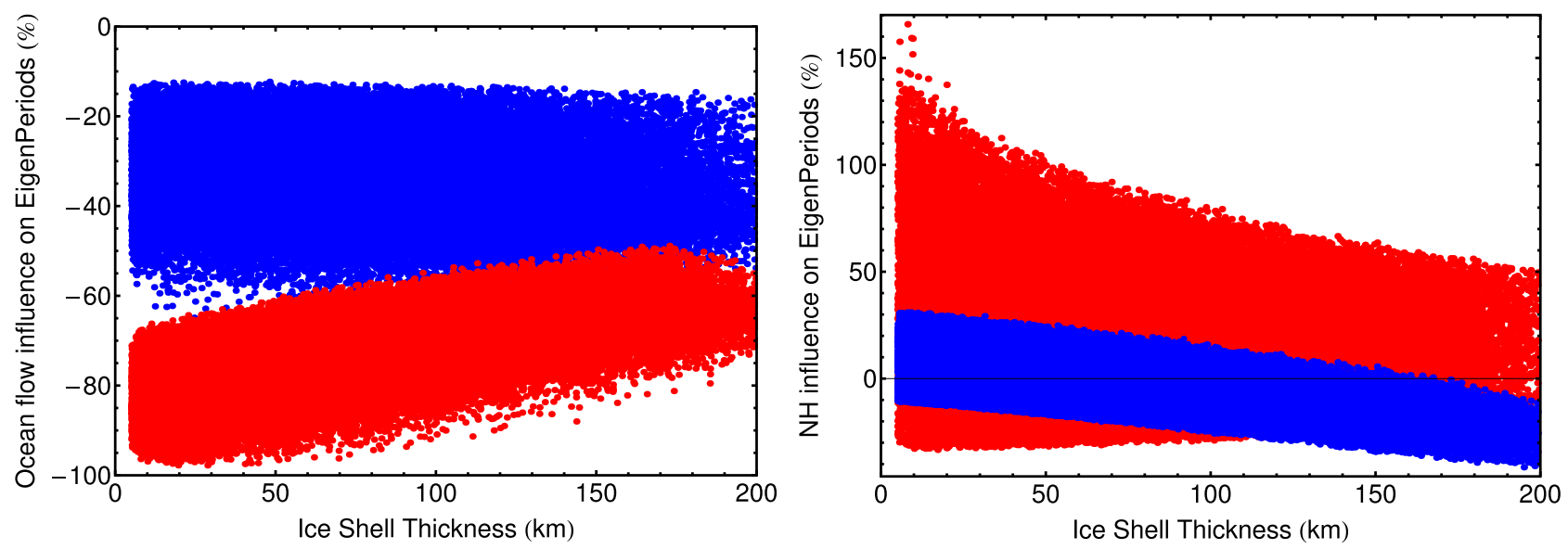

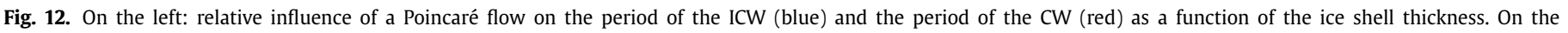

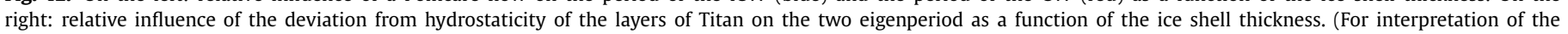
references to color in this figure legend, the reader is referred to the web version of this article.)

motion from the pole of the BF of the shell for the shell and the ocean, and from the pole of the BF of the interior for the interior. These polar offsets are given by

$\omega_{s}^{x, \text { off }}=n \frac{K_{43} K_{65} h^{x}(0)}{K_{25}\left(K_{43} K_{61}-K_{41} K_{63}\right)+K_{65}\left(K_{23} K_{41}-K_{21} K_{43}\right)}$
$\omega_{s}^{y, \text { off }}=n \frac{K_{34} K_{56} h^{y}(0)}{K_{16}\left(K_{32} K_{54}-K_{34} K_{52}\right)+K_{56}\left(K_{12} K_{34}-K_{14} K_{32}\right)}$

$\omega_{o}^{x, \text { off }}=\omega_{s}^{X, \text { off }}$

$\omega_{o}^{y, \text { off }}=\omega_{s}^{y, \text { off }}$

$\omega_{i}^{x, \text { off }}=n \frac{\left(K_{41} K_{63}-K_{43} K_{61}\right) h^{x}(0)}{K_{25}\left(K_{43} K_{61}-K_{41} K_{63}\right)+K_{65}\left(K_{23} K_{41}-K_{21} K_{43}\right)}$

$\omega_{i}^{y, \text { off }}=n \frac{\left(K_{32} K_{54}-K_{34} K_{52}\right) h^{y}(0)}{K_{16}\left(K_{32} K_{54}-K_{34} K_{52}\right)+K_{56}\left(K_{12} K_{34}-K_{14} K_{32}\right)}$.

The components of the polar offset are proportional to the constant terms of the atmospheric/ocean angular momentum (see Eq. (40)). Polar motion trajectories circle about the secular position $(R / n)\left(\omega_{s}^{x, \text { off }}, \omega_{s}^{y, \text { off }}\right)$ which is not the $z$-axis of the BF of the shell. The polar offset is of the order of -100 to $100 \mathrm{~m}$ in the $x$ direction and of the order of -2500 to $-4000 \mathrm{~m}$ in the $y$ direction (see Fig. 13). The $x$-component of the polar offset is either positive for positive value of $h^{x}(0)$ or negative for negative values of $h^{x}(0)$ while the $y$-component is always negative as $h^{y}(0)$ is negative for all the interior models. The Poincaré flow in the subsurface ocean has a large influence on the $y$-component of the polar offset, with a polar offset increasing from $-500 \mathrm{~m}$ without Poincaré flow to $-4000 \mathrm{~m}$ for thin shells (see right panel of Fig. 13).

The deviation from hydrostaticity has a smaller effect on the $y$ component of the polar offset but can be the most important effect on the $x$-component and for thin shells.

In comparison with the $z$-component of the atmospheric torque (see top panel in Fig. 5), the $x$ and $y$-components of the atmospheric torque present a slower decrease with decreasing atmospheric forcing period (see top panel of Fig. 14). As a result, we expect that the ter-annual and 1/4-annual forcing could more easily have a significant influence on the polar motion than on the LOD variations. The $y$-component of the atmospheric torque has a main annual amplitude of $1.37 \times 10^{17} \mathrm{Nm}$. The second and third largest forcings occur at $1 / 4$-annual $(7.4 \mathrm{y})$ and semi-annual (14.7 y) periods, with an amplitude of $5.83 \times 10^{16} \mathrm{Nm}$ and $4.59 \times 10^{16} \mathrm{Nm}$, respectively. The $x$-component of the torque has a main semi-annual amplitude of $1.15 \times 10^{17} \mathrm{Nm}$. The second and third largest forcings occur at ter-annual ( $9.8 \mathrm{y}$ ) and annual periods, with an amplitude of $8.19 \times 10^{16} \mathrm{Nm}$ and $6.93 \times 10^{16} \mathrm{Nm}$, respectively.

The polar motion amplitudes at the different atmospheric frequencies $\varpi$ are of the following form

$$
\Omega_{s / 0 / i}^{x / y, \cos / \sin }(\varpi)=\frac{\tilde{\Omega}_{s / 0 / i}^{x / y, \cos / \sin }(\varpi)}{\tilde{A}_{s} A_{0} \tilde{A}_{i} \tilde{B}_{s} B_{0} \tilde{B}_{i}\left(\varpi^{2}-\sigma_{1}^{2}\right)\left(\varpi^{2}-\sigma_{2}^{2}\right)\left(\varpi^{2}-\sigma_{3}^{2}\right)},
$$

but the expressions for the $\tilde{\Omega}_{s / 0 / i}^{x / y, \cos / \sin }$ coefficients are too long to be given explicitly here. The polar motion amplitudes depend on the value of the NDFW ( $\sigma_{3}$ in Eq. (67)) but as the forcing frequencies $\varpi$ are far from the diurnal frequency, the NDFW has no significant influence on the polar motion amplitudes. Fig. 14 presents the different polar motion amplitudes at all forcing frequencies $\varpi$ retained here (i.e. corresponding to periods larger than 10 Titan's day) for one specific interior model of Titan with an ice shell thickness of $200 \mathrm{~km}$. For this ice shell thickness, the amplitude of the polar motion of the shell is maximal at the annual period with an amplitude larger than $100 \mathrm{~m}$.

In order to study the amplitude of the polar motion of the shell as a function of the thickness of the ice shell, we consider the four main atmospheric forcing periods (see Fig. 15). The amplitudes present resonant amplifications as the atmospheric forcing periods can be close to the CW period (see Fig. 11). For ice shells thicker than $50 \mathrm{~km}$, the amplitude is maximal at the annual period. The amplitude at annual period is resonantly amplified for an ice shell thickness of about $25 \mathrm{~km}$ to $100 \mathrm{~km}$ depending on the internal structure. For thinner ice shells, the polar motion amplitudes are amplified at shorter periods. For instance, the semi-annual amplitude becomes dominant for ice shell thickness between $15 \mathrm{~km}$ and $35 \mathrm{~km}$ and the ter-annual for ice shell thickness between $10 \mathrm{~km}$ and $20 \mathrm{~km}$. The Poincare flow in the subsurface ocean strongly influences the polar motion amplitudes as it lengthens the resonant eigenperiod and brings it closer to the main atmospheric periods (see Fig. 16). In comparison with the influence of a Poincare flow, the deviation of the shape of the internal boundaries and surface from hydrostaticity has a smaller impact on the polar motion amplitudes for ice shells thicker than $100 \mathrm{~km}$. It strongly influences 

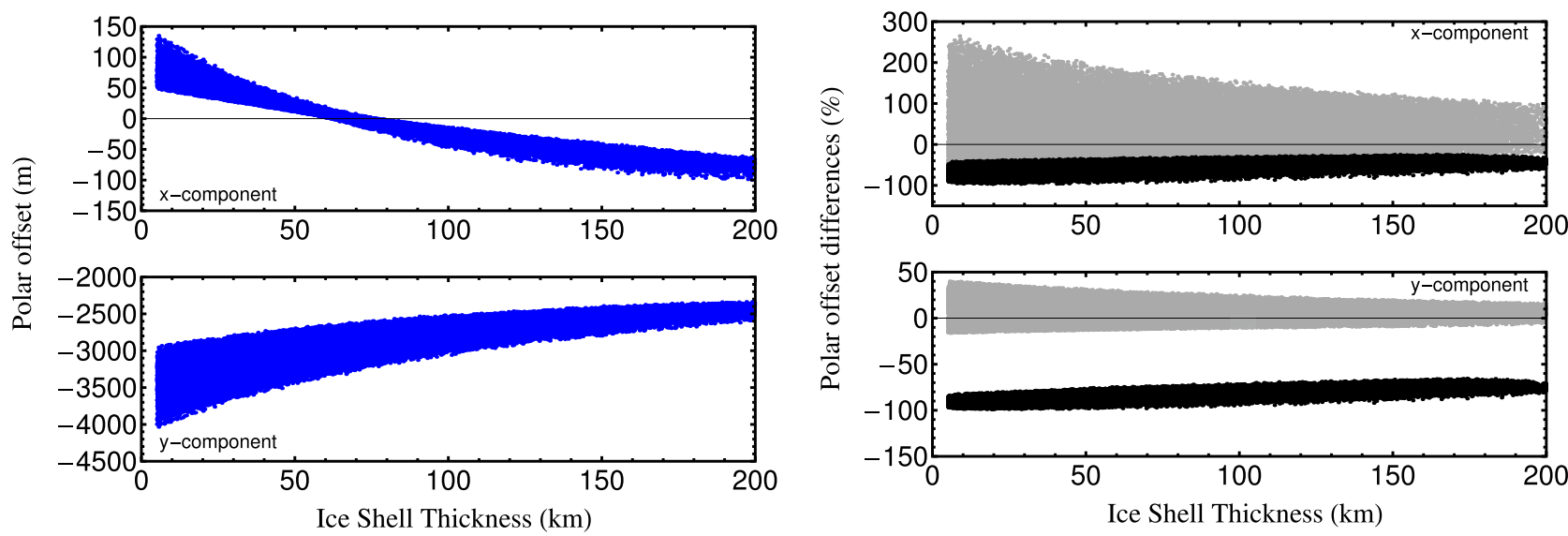

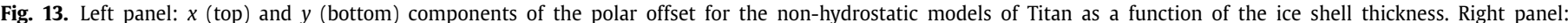
Influence (in \%) of the non-hydrostatic interior of Titan (gray) and of the Poincare flow in the subsurface ocean (black) on the polar offset.
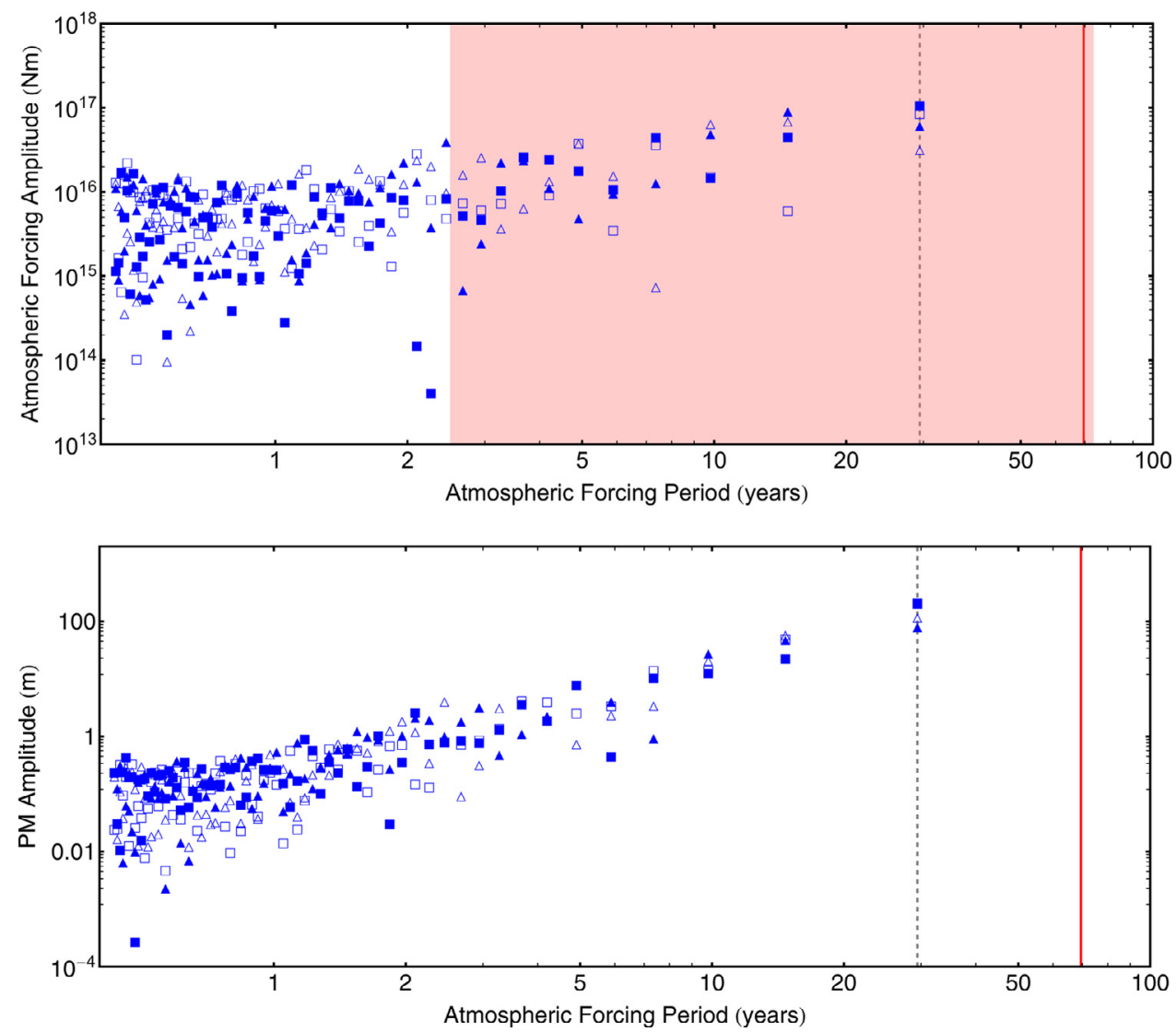

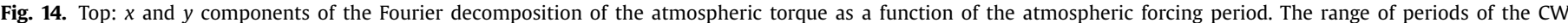

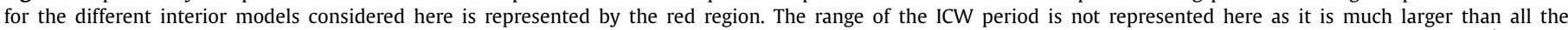

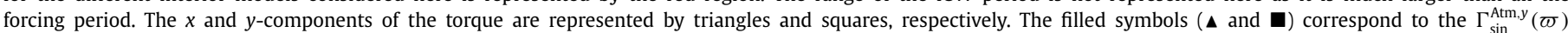

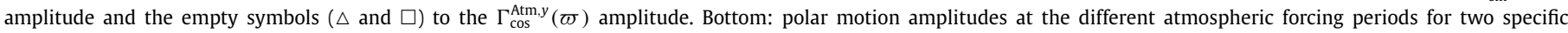

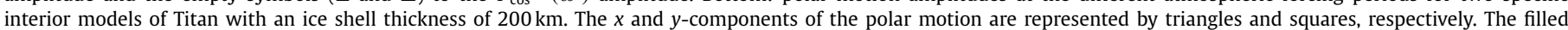

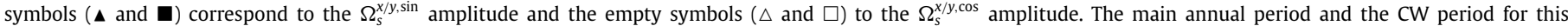

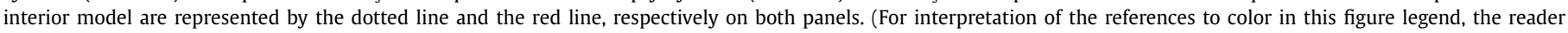
is referred to the web version of this article.)

the polar motion amplitudes for interior models with ice shells thinner than $50 \mathrm{~km}$ as it shortens the resonant eigenperiod.

To illustrate the behavior of polar motion as a function of time and to demonstrate that it depends sensitively on Titan's interior structure, we present in Fig. 17 the polar motion trajectories for particular interior models of Titan with an ice shell density of $1000 \mathrm{~kg} / \mathrm{m}^{3}$ and ice shell thicknesses ranging from $200 \mathrm{~km}$ down to $50 \mathrm{~km}$. For thick ice shells, the main contribution to the polar motion is annual because of the absence of resonances, but there is also a significant semi-annual contribution. The polar motion follows an anticlockwise trajectory with a somewhat larger amplitude in the $y$ direction due to the larger $y$-component of the atmospheric forcing at the main annual period. Polar motion amplitudes increase with decreasing ice shell thicknesses, but the semi-annual 

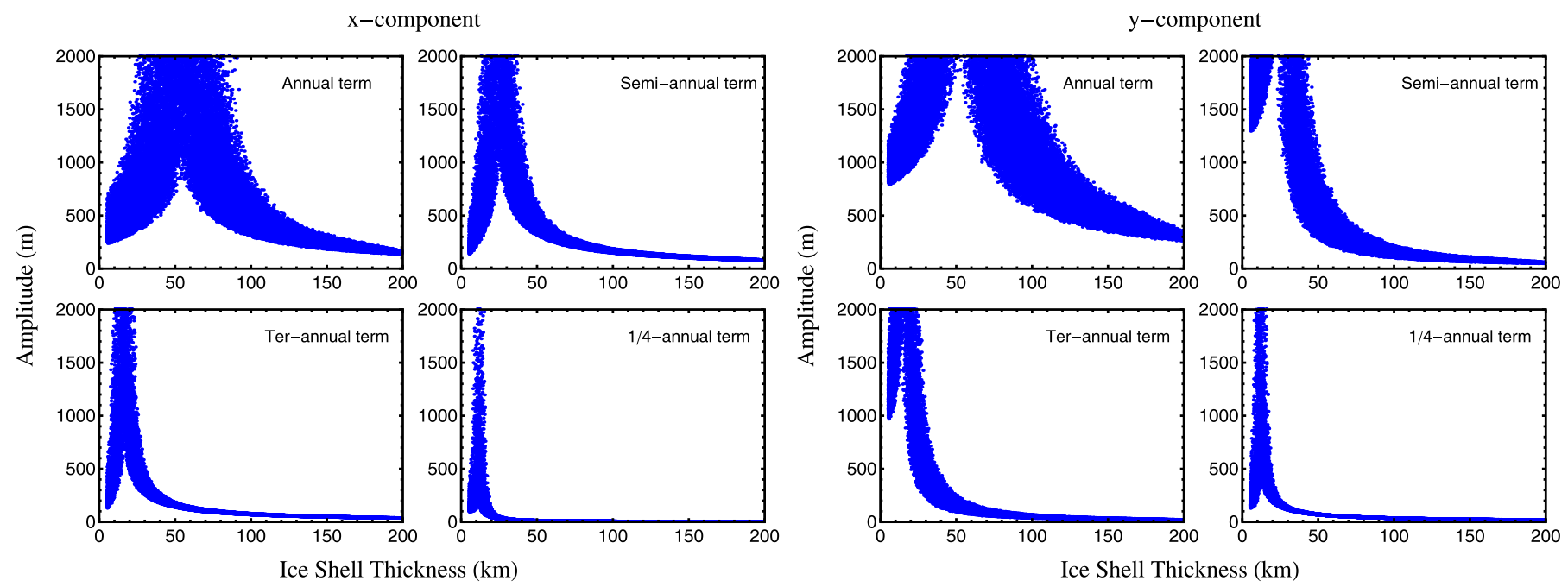

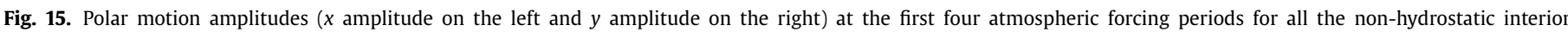
models of Titan.
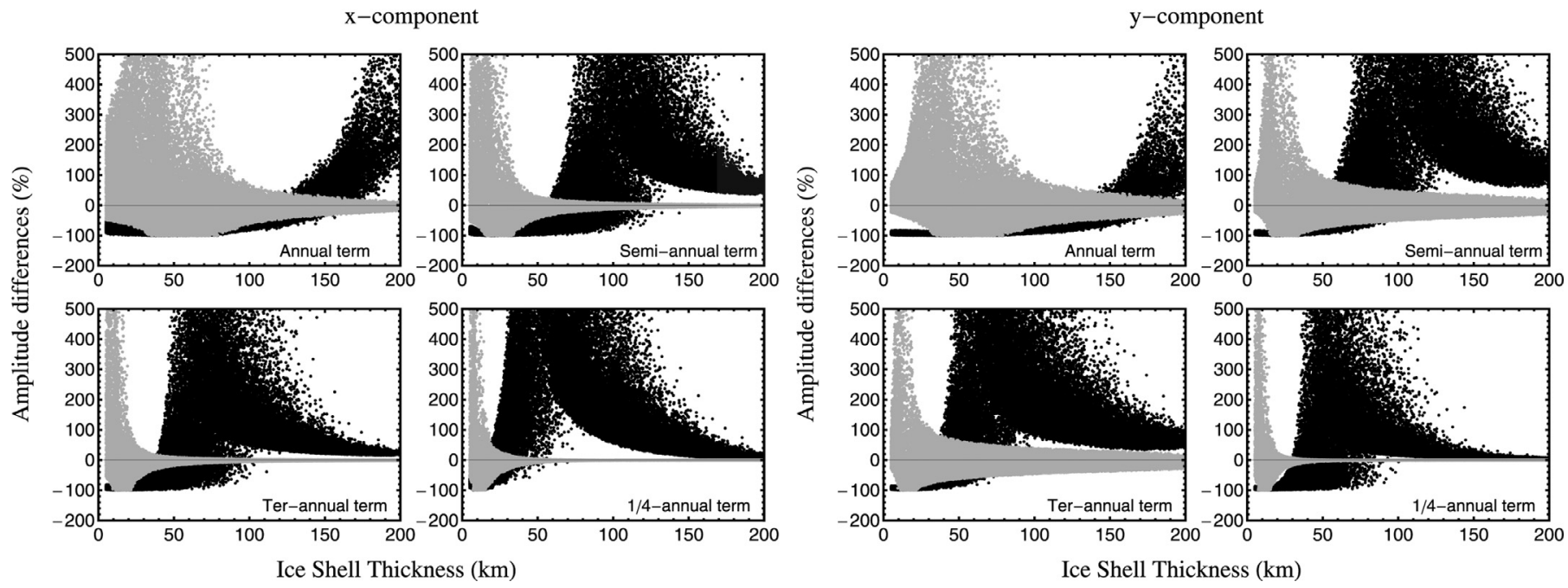

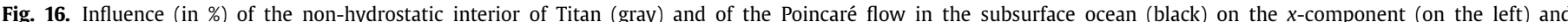
$y$-component (on the right) of the polar motion and for the first four atmospheric forcing periods.

increases slower that the annual amplitude. The polar motion amplitude is of the order of $500 \mathrm{~m}$ in the $y$ direction and $200 \mathrm{~m}$ in the $x$ direction for an ice shell thickness of $200 \mathrm{~km}$ and larger than $5 \mathrm{~km}$ for an ice shell thickness of $50 \mathrm{~km}$.

\section{Discussion}

\subsection{Influence of shell rigidity and viscosity}

In the preceding sections, we fixed the rigidity of the ice shell to $3.3 \mathrm{GPa}$. We here investigate the influence of the rheology of the ice shell on the results presented in these sections. We consider a Maxwell rheology model to describe the viscoelastic properties of the ice shell (see e.g. Sabadini and Vermeersen, 2004). In this model, the effective rigidity $\tilde{\mu}$ of the ice shell is given by

$\tilde{\mu}=\mu\left(\frac{i \varpi}{i \varpi+\frac{\mu}{\eta}}\right)$,

where $i$ is the imaginary number, $\mu$ is the real rigidity of the ice shell and $\eta$ its viscosity. We divide the ice shell into two layers: a convective bottom layer and a conductive top layer. We here only consider interior models with ice shell thicker than $50 \mathrm{~km}$, as thinner ice shells are excluded from recent studies of Schumann resonance in the atmosphere of Titan (Béghin et al., 2012) or of the low-degree gravity and topography of Titan (Hemingway et al., 2013). We set the thickness of the top part of the ice shell equal to $40 \mathrm{~km}$, the minimal elastic thickness obtained by Hemingway et al. (2013). The rigidity of the interior has no significant influence on our results because the tides of the interior are much smaller than these of the shell and almost do not affect the deformations of the shell. We fix the rigidity of the convective bottom part of the ice shell to $3.3 \mathrm{GPa}$ and consider rigidities of the top part of the ice shell ranging from $1 \mathrm{GPa}$ to $5 \mathrm{GPa}$. This range includes the $2 \mathrm{GPa}$ rigidity value obtained by Cole and Durell (1995), as well as the $3.5 \mathrm{GPa}$ value obtained by Helgerud et al. (2009). As the viscosity decreases with increasing temperature, we choose here a viscosity ranging between $10^{13} \mathrm{~Pa} \mathrm{~s}$ and $10^{16} \mathrm{~Pa} \mathrm{~s}$ for the hotter conductive bottom part of the ice shell (see e.g. Barr and Showman, 2009) and a viscosity of $10^{16}$ Pa $s$ for the cold top part of the ice shell. We tested that this and higher viscosity values for the top part lead to essentially the same rotation results as for an elastic top layer. 

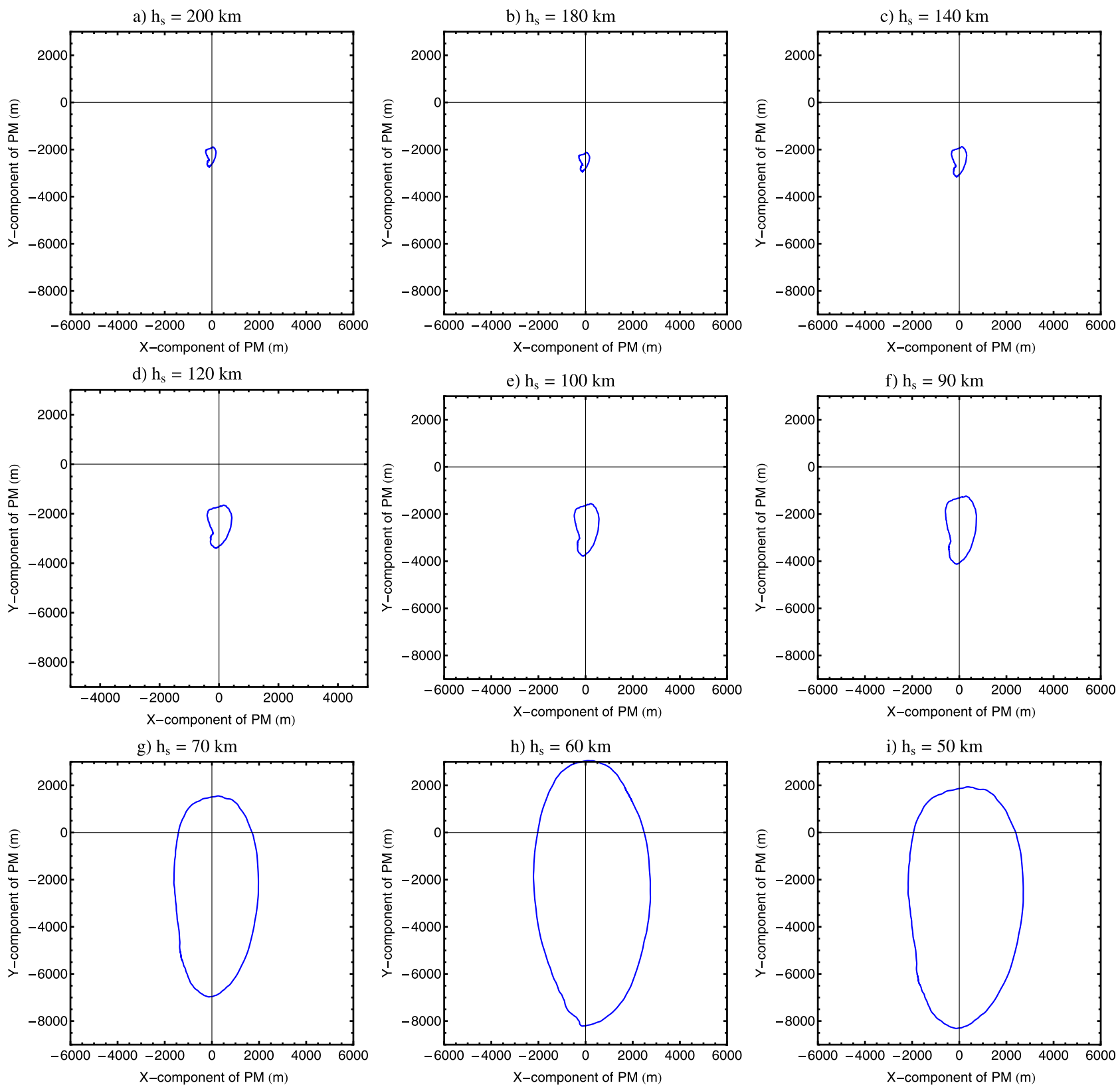

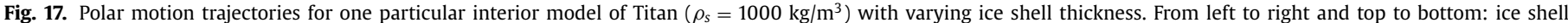
thickness of $200 \mathrm{~km}, 180 \mathrm{~km}, 140 \mathrm{~km}, 120 \mathrm{~km}, 100 \mathrm{~km}, 90 \mathrm{~km}, 70 \mathrm{~km}, 60 \mathrm{~km}$ and $50 \mathrm{~km}$.

For all the interior models with ice shell thicker than $50 \mathrm{~km}$, the amplitudes of the diurnal libration linearly decrease with increasing ice shell rigidity and decrease with increasing ice shell viscosity. This is similar to results presented by Jara-Orué and Vermeersen (2014) or Van Hoolst et al. (2016) who use the absolute values of the libration amplitudes. The maximal variation of the libration amplitude induced by a variation of the top ice shell rigidity from 1 to $5 \mathrm{GPa}$ or by a variation of the bottom ice shell viscosity from $10^{13}$ to $10^{16}$ is below $20 \mathrm{~m}$, well below the precision of possible observations (see Section 5.2). The influence of the ice shell rigidity and viscosity on the libration amplitudes can therefore be neglected at the precision level considered here.

The variation of the ice shell rigidity or ice shell viscosity has also only a small influence on the main semi-annual LOD amplitudes (smaller than $0.5 \%$ and $5 \%$ for variations of the ice shell rigidity and ice shell viscosity, respectively) and on the main annual polar motion (smaller than $0.5 \%$ and $2 \%$ for variations of ice shell rigidity and ice shell viscosity, respectively). The polar offset is almost constant with varying ice shell rigidity or viscosity, with a variation smaller than $1 \mathrm{~m}$. Close to a resonance, as can be the case for the LOD or for the polar motion, a small variation can result in an amplitude variation larger than the precision of possible observations. However, the uncertainties in LOD and polar motion amplitudes due to the ice shell rigidity or viscosity are smaller than uncertainties due to other interior parameters (such as layer' densities and radii). We can therefore consider that ice shell rigidity and ice shell viscosity variations do not affect the range of the LOD and polar motion amplitudes.

\subsection{Comparison with observations}

\subsubsection{Rotation rate}

The rotation of Titan can be studied by comparing the position of different surface landmarks that appear on two (or more) 


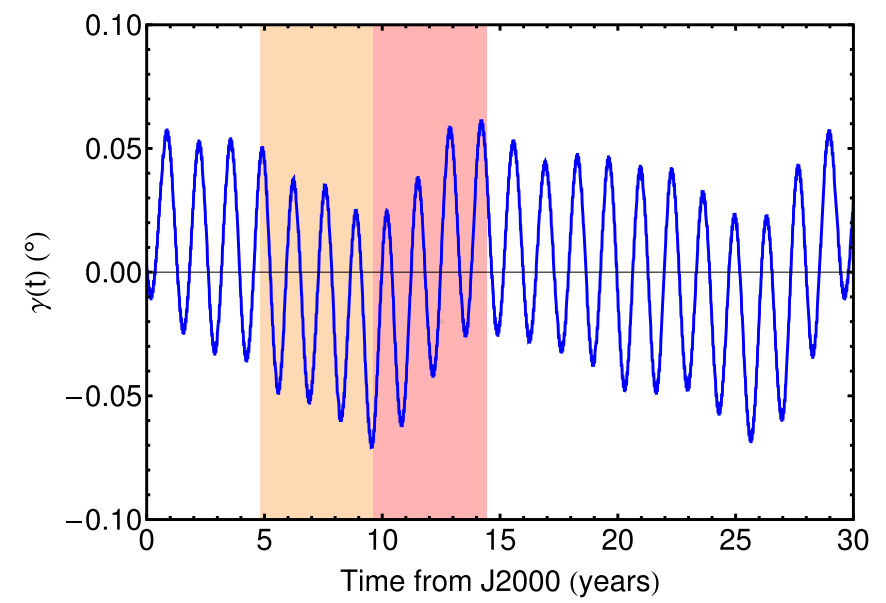

Fig. 18. LOD variations $\gamma_{s}(t)$ during one saturnian year and for one particular interior model of Titan with an ice shell thickness of $100 \mathrm{~km}$. The orange region corresponds to the 61 first flybys of Titan during the period 2004-2009 used by Meriggiola et al. (2016) and the red region to a period of time of the same length of that used by Meriggiola et al. (2016) but starting from the 61st flyby of Titan. (For interpretation of the references to color in this figure legend, the reader is referred to the web version of this article.)

RADAR images taken during different flybys of Titan by the Cassini probe. The precision of a position on one RADAR image taken by the Cassini probe is of the order of $0.9-1.3 \mathrm{~km}$ (corresponding to about 6-8 pixels on the image, Meriggiola et al., 2016). As the amplitudes of the diurnal libration, of the LOD variations and of the polar motion can be larger than this precision for some interior models, we could expect to detect these rotation variations if Titan's interior is close enough to some of those interior models.

The deviation of the layer's shape from hydrostatic shapes strongly influences the diurnal libration amplitude of the shell, with an amplitude that can be more than thirty times larger than for an equivalent hydrostatic model. However, the amplitude of the diurnal libration is larger than the image precision only for a few interior models with ice shells thinner than $15 \mathrm{~km}$ (see Fig. 9), a value that is not consistent with the low-degree gravity and topography data (Hemingway et al., 2013) and with the measurements of low frequency waves and conductivity of the atmosphere of Titan (Béghin et al., 2012). At long period, the LOD variations are somewhat diminished by the deviation of the layers' shape from hydrostatic shapes. However, the amplitude of the LOD variations is larger than the image precision for most ice shell thicknesses (see Fig. 8). Moreover, due to the resonance with the two librational eigenperiods, the LOD present large variations with timescales at the order of 1-4 years.

Using 160 RADAR images of the surface of Titan taken by the Cassini probe during its 61 first flybys between 2004 and 2009, Meriggiola et al. (2016) have determined a mean nonsynchronous rotation (NSR) of about $-0.024^{\circ} \pm 0.018^{\circ} /$ year (with a $1-\sigma$ error), close to a synchronous value of the spin rate rotation. From Fig. 8, we see that the LOD variations show a decreasing trend in the period of time of the flybys studied by Meriggiola et al. (2016) (see orange region in Fig. 8), as this period of time happens before the Northern vernal equinox (11 August 2009). This can also be seen in Fig. 18 where the LOD variations $\gamma_{s}$ of one particular interior model with ice shell thickness of $100 \mathrm{~km}$ is presented as a function of time. For this particular model, the mean NSR over the $2004-2009$ period is $-0.0242^{\circ}$ year, equal to the NSR value obtained by Meriggiola et al. (2016). The mean NSR $\dot{\gamma}_{s}$ over the 2004-2009 period is presented in Fig. 19 for all the interior models considered here. It can be larger than the maximal value of about $0.013 \%$ year obtained by Van Hoolst et al. (2013) as

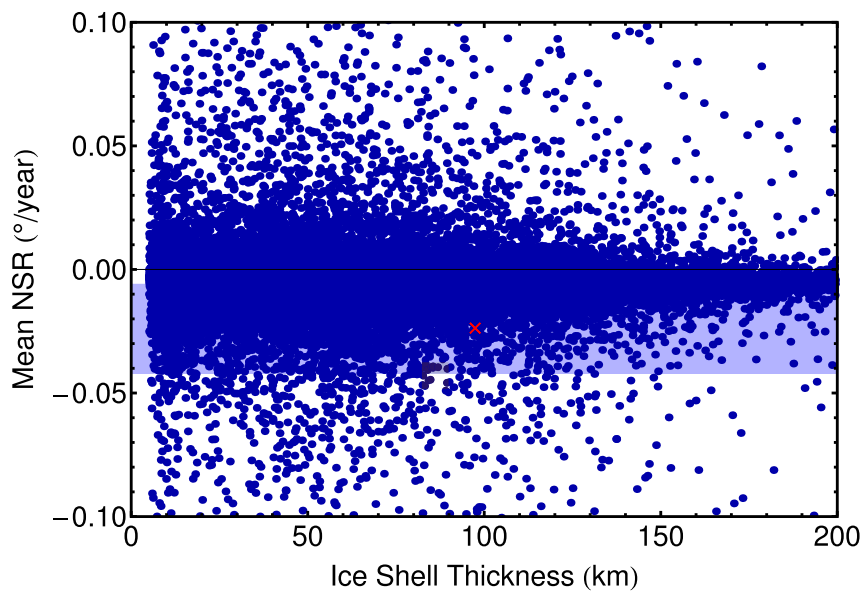

Fig. 19. Mean value of the non-synchronous rotation rate $\gamma_{s}^{\prime}(t)$ during the period 2004-2009 corresponding to the 61 first flybys of Titan by Cassini. The mean value and uncertainty of the NSR obtained by Meriggiola et al. (2016) is represented in light blue. The red cross corresponds to the mean LOD variations of the interior model presented in Fig. (18). (For interpretation of the references to color in this figure legend, the reader is referred to the web version of this article.)

we here include the shorter periods of the LOD variations that can have a large amplitude (see blue curve in Fig. 18). The mean NSR over the 2004-2009 period is negative for a large part of the interior models (87\% of the interior models). Some interior models are characterized by a positive value of the mean NSR over the 2004-2009 period due to the variations of the LOD variations at short periods of about one to five years. The mean NSR over the 2004-2009 period is compatible with the observation of Meriggiola et al. (2016) for $47 \%$ for all the interior models and $50 \%$ for interior models with an ice shell thicker than $50 \mathrm{~km}$. The value of the NSR from Meriggiola et al. (2016) could therefore be interpreted as an observation of the LOD variations due to the atmosphere of Titan during that period of time while the large uncertainty on this value could be due to the LOD variations at short periods. Unfortunately, the interior structure of Titan cannot be constrained from this observation (see Fig. 19 for the ice shell thickness; other interior parameters have been tested but do not present any trend). If our interpretation is correct, we predict that the NSR in a period of time of the same length of that used by Meriggiola et al. (2016) but starting from the 61st flyby (2009) of Titan is very likely positive (see red region in Fig. 18). In that period of time, the mean NSR is positive for $91 \%$ of the interior models considered here and for $95 \%$ of the interior models compatible with the observed NSR over 2004-2009. For these interior models, the mean value of the NSR during that period of time is $\mathrm{NSR}=0.0080 \pm 0.0391^{\circ}$ /year. The study of the Cassini RADAR data obtained during the Prime and Extended Missions needs to be continued in order to see possible variations of the NSR between the Prime and the Extended Missions. It would also be useful to see if short periods oscillations of the NSR could also be identified in the Cassini data.

\subsubsection{Polar motion}

In principle, an analysis of Cassini RADAR observations over the entire mission duration could determine the amplitude of the polar motion at annual period. For interior models with an ice shell thicker than $50 \mathrm{~km}$, the polar motion is mainly annual with a significant semi-annual term. The annual amplitude of the polar motion is larger than the detection level for ice shell thicknesses thinner than $100 \mathrm{~km}$ (see Fig. 15). If a significant other period could be detected, the ice shell thickness could be constrained by combining these polar motion amplitudes. 


\section{Conclusion}

Using the Euler-Liouville equations, we have studied the rotation rate variations and polar motion of a non-hydrostatic Titan. Rotation rate variations are due to the gravitational torque exerted by Saturn (diurnal librations) or the atmosphere of Titan (LOD variations).

In comparison with the results obtained by Van Hoolst et al. (2013) for hydrostatic models of Titan, the amplitude of the main semi-annual LOD variations is somewhat diminished by the deviation from hydrostaticity of the layers of Titan. The amplitudes of the LOD variations at shorter periods (shorter than 5 years) can be strongly increased due to a resonance amplification with a librational eigenperiod.

In comparison with uncertainties related to other internal parameters, a variation of the rigidity and viscosity of the ice shell has only a weak influence on the amplitude of the LOD and polar motion. The variation of the amplitude of the diurnal librations due to a variation of ice shell rigidity or ice shell viscosity is smaller than $20 \mathrm{~m}$, well below the detection level, so that the influence of the ice shell rigidity and ice shell viscosity can be neglected.

For about $50 \%$ of all interior models with ice shell thicker than $50 \mathrm{~km}$, the LOD variations between 2004 and 2009 present a main decreasing trend that is compatible with the mean NSR computed by Meriggiola et al. (2016) using RADAR images taken by Cassini during that period. We therefore consider that this mean NSR can be interpreted as an observation of the LOD variations between 2004 and 2009. Unfortunately, it is not possible to use the observation of Meriggiola et al. (2016) to constrain the interior structure of Titan as the mean NSR does not present any trend with the different interior model parameters.

The amplitude of the diurnal librations is strongly influenced by the deviation from the hydrostatic shape of the shell of Titan. While the amplitudes of the diurnal librations for an hydrostatic Titan only weakly depend on the interior structure, the amplitudes of the diurnal librations for interior models of Titan can largely increase as the external gravitational torque exerted by Saturn increases due to the deviation from hydrostaticity of the shape of the shell. The libration amplitude can be larger than $1 \mathrm{~km}$ for thin ice shell. For some interior models with a strong deviation from hydrostaticity, the tidal torque between the tidal and static bulges of the shell and interior can even become dominant, leading to librations in the opposite direction.

Polar motion of Titan is mainly forced by the atmosphere of Titan. We here only considered the long-period variations ( $>10$ Titan days) of the polar motion which can be studied decoupled from the study of the nutations.

For interior models with ice shell thicker than $50 \mathrm{~km}$, the polar motion has a main annual period where the atmospheric forcing is largest. Its amplitude can be amplified by a resonance with the $\mathrm{CW}$ and increases from $500 \mathrm{~m}$ to $5 \mathrm{~km}$ with decreasing ice shell thickness from $200 \mathrm{~km}$ to $50 \mathrm{~km}$. The polar motion is strongly influenced by the flow in the subsurface ocean. Deviation from the hydrostatic shapes of the layers of Titan also has a significant influence on the polar motion for interior models close to a resonance.

\section{Acknowledgments}

This work was financially supported by the Belgian PRODEX program managed by the European Space Agency (grant no. 4000120791) in collaboration with the Belgian Federal Science Policy Office. We thank the Editor, Marie Yseboodt, Benoît Noyelles and the second anonymous reviewer for their helpful comments and suggestions that have helped to improve our paper.

\section{Appendix A. Non-hydrostatic flattenings}

The total gravitational, tidal and centrifugal potential at the surface of the layer $k$ is given by

$\Phi_{k}\left(r_{k}\right)=\frac{G M\left(R_{k}\right)}{r_{k}}+\frac{1}{3} n^{2} r_{k}^{2}+\Phi_{k}^{20}\left(r_{k}\right) R_{20}+\Phi_{k}^{22}\left(r_{k}\right) R_{22}$

where $M(r)$ represents the total mass of the materials located under the mean radius $r$. For the mantle, we have

$$
\begin{aligned}
\Phi_{m}^{20}\left(R_{m}\right)= & -\frac{5}{6} n^{2} R_{m}^{2}-\frac{8 \pi G}{15} R_{m}^{2}\left\{\left[\alpha_{s} \rho_{s}+\alpha_{o}\left(\rho_{o}-\rho_{s}\right)-\alpha_{m} \rho_{o}\right]\right. \\
& \left.+\alpha_{m} \rho_{m}+\alpha_{c}\left(\rho_{c}-\rho_{m}\right) \frac{R_{c}^{5}}{R_{m}^{5}}\right\} \\
\Phi_{m}^{22}\left(R_{m}\right)= & \frac{1}{4} n^{2} R_{m}^{2}+\frac{2 \pi G}{15} R_{m}^{2}\left\{\left[\beta_{s} \rho_{s}+\beta_{o}\left(\rho_{o}-\rho_{s}\right)-\beta_{m} \rho_{o}\right]\right. \\
& \left.+\beta_{m} \rho_{m}+\beta_{c}\left(\rho_{c}-\rho_{m}\right) \frac{R_{c}^{5}}{R_{m}^{5}}\right\}
\end{aligned}
$$

and for the core

$$
\begin{aligned}
\Phi_{c}^{20}\left(R_{c}\right)= & -\frac{5}{6} n^{2} R_{c}^{2}-\frac{8 \pi G}{15} R_{c}^{2}\left[\alpha_{s} \rho_{s}+\alpha_{o}\left(\rho_{o}-\rho_{s}\right)\right. \\
& \left.+\alpha_{m}\left(\rho_{m}-\rho_{o}\right)+\alpha_{c}\left(\rho_{c}-\rho_{m}\right)\right] \\
\Phi_{c}^{22}\left(R_{c}\right)= & \frac{1}{4} n^{2} R_{c}^{2}+\frac{2 \pi G}{15} R_{c}^{2}\left[\beta_{s} \rho_{s}+\beta_{o}\left(\rho_{o}-\rho_{s}\right)\right. \\
& \left.+\beta_{m}\left(\rho_{m}-\rho_{o}\right)+\beta_{c}\left(\rho_{c}-\rho_{m}\right)\right] .
\end{aligned}
$$

The surface of the mantle and the core being an equipotential surface, $\Phi_{k}\left(R_{k}\right)$ (for $k=c$ and $k=m$ ) must be constant at these surfaces and we find the equations for the mantle flattenings $\alpha_{k}$ and $\beta_{k}$ (see Baland et al., 2014, for more details)

$-\frac{2}{3} \alpha_{k} R_{k}=\frac{\Phi_{k}^{20}\left(R_{k}\right)}{g\left(R_{k}\right)}$

$\frac{1}{6} \beta_{k} R_{k}=\frac{\Phi_{k}^{22}\left(R_{k}\right)}{g\left(R_{k}\right)}$,

where $g\left(R_{k}\right)$ is the gravitational acceleration due to masses located under the mean radius $R_{k}$.

\section{Appendix B. Inertia products}

Titan is deformed due to the tides and the changes in the rotation which modify the centrifugal potential. Both the centrifugal potential $V_{\text {per, } k}^{\text {cent }}(\vec{r})$ and the tidal potential $V_{\text {per, } k}^{\mathrm{t}}(\vec{r})$ are periodically affected by the librations, LOD variations and polar motion. The tidal potential is also affected by the obliquity (see Coyette et al., 2016).

Using the definition of the $k_{2}$ Love number generalized for the different layers of Titan (e.g. Coyette et al., 2016), the periodic external additional gravitational potential at a distance $r$ from the mass center of the satellite can be written, for each layer $k$, as

$V_{\mathrm{per}, k}^{\prime}(r)=\left(\frac{R}{r}\right)^{3} k_{2, k}\left[V_{\mathrm{per}, k}^{\mathrm{cent}}(R)+V_{\mathrm{per}, k}^{\mathrm{t}}(R)\right]$

By equaling the right-hand side of Eq. (B.1) with the expression for the degree-two gravitational potential of Titan at the surface of Titan and due to the layer $k$

$$
\begin{aligned}
\Phi(r, \lambda, \theta)= & -\frac{G M_{T}}{r}\left(\frac{R}{r}\right)^{2} \sum_{m}^{2}\left[C_{k}^{2 m} \cos (m \lambda)\right. \\
& \left.+S_{k}^{2 m} \sin (m \lambda)\right] P_{2}^{m}(\cos \varphi),
\end{aligned}
$$


and using the existing relations between the degree two coefficients $C_{\text {per }}^{l m}$ and the incremental inertia products $c^{l m}$, it is possible to find that (see Coyette et al., 2016, for more details)

$c_{k}^{12}=-2 k_{2, k} q_{r} M_{T} R^{2}\left(e \sin M-\frac{\gamma_{k}}{2}\right)$

$c_{k}^{13}=k_{2, k} q_{r} M_{T} R^{2}\left(\frac{4}{3 n} \omega_{k}^{x}-\eta_{k} \sin (M+\omega)\right)$

$c_{k}^{23}=k_{2, k} q_{r} M_{T} R^{2} \frac{\omega_{k}^{y}}{3 n}$,

$c_{k}^{33}=k_{2, k} R^{2}\left(q_{r} M_{T} e \cos M+4 n R^{3} \frac{\dot{\gamma}_{k}}{9 G}\right)$,

where $q_{r}=\left(n^{2} R^{3}\right) /\left(G M_{T}\right)$.

\section{Appendix C. Internal gravitational torques between layers}

The internal gravitational torque exerted by an outer layer $O$ on an inner layer $I$ is given by

$\vec{\Gamma}_{g, I}=\int_{V_{I}} \rho_{I} \vec{r} \times \vec{\nabla} \Phi(\vec{r}) d V$,

where $V_{I}$ and $\rho_{I}$ is the interior volume and density, respectively, and $\Phi(\vec{r})$ is the gravitational potential due to the other outer layer 0 .

We express the gravitational potential $\Phi(\vec{r})$ and the vector position $\vec{r}$ as a series of Legendre polynomials

$$
\begin{aligned}
\Phi(\vec{r})= & r^{2}\left(\left(\phi_{20}+\delta \phi_{20}\right) R_{20}+\delta \phi_{21}^{\cos } R_{21}+\delta \phi_{21}^{\sin } S_{21}\right. \\
& \left.+\left(\phi_{22}+\delta \phi_{22}^{r}\right) R_{22}+\delta \phi_{22}^{l} S_{22}\right)
\end{aligned}
$$

$$
\begin{aligned}
r= & R\left(1-\frac{2}{3} \alpha_{I}\left[\left(1+\delta h_{I}^{\perp}\right) R_{20}+\delta h_{I}^{\mathrm{obl}, \cos } R_{21}+\delta h_{I}^{\mathrm{obl}, \sin } S_{21}\right]\right. \\
& \left.+\frac{\beta_{I}}{6}\left[\left(1+\delta h_{I}^{r}\right) R_{22}+\delta h_{I}^{l} S_{22}\right]\right),
\end{aligned}
$$

where the terms designated by $\delta$ are one order of magnitude smaller than the other terms. The different terms $\phi_{l m}$ are given by

$\phi_{20}=-\frac{8 \pi G}{15} \int_{0} \rho(r) \frac{\partial \alpha(r)}{\partial r} d r$

$\delta \phi_{20}=-\frac{8 \pi G}{15} \int_{0} \rho(r) \frac{\partial \alpha(r) \delta h_{j}^{\perp}}{\partial r} d r$

$\delta \phi_{21}^{\mathrm{sin}}=-\frac{8 \pi G}{15} \int_{0} \rho(r) \frac{\partial \alpha(r) \delta h_{j}^{\mathrm{obl}, \mathrm{sin}}}{\partial r} d r$

$\delta \phi_{21}^{\cos }=-\frac{8 \pi G}{15} \int_{0} \rho(r) \frac{\partial \alpha(r) \delta h_{j}^{\mathrm{obl}, \cos }}{\partial r} d r$

$\phi_{22}=\frac{2 \pi G}{15} \int_{0} \rho(r) \frac{\partial \beta(r)}{\partial r} d r$

$\delta \phi_{22}^{r}=\frac{2 \pi G}{15} \int_{0} \rho(r) \frac{\partial \beta(r) \delta h_{j}^{r}}{\partial r} d r$

$\delta \phi_{22}^{l}=\frac{2 \pi G}{15} \int_{0} \rho(r) \frac{\partial \beta(r) \delta h_{j}^{l}}{\partial r} d r$.
As Szeto and Xu (1997) (see also Coyette et al., 2016), we express the gravitational potential $\Phi(\vec{r})$ as a function of the coordinates related to the $\mathrm{BF}$ of the interior by using the following transformation between a vector expressed in the $\mathrm{BF}$ of the interior and the $\mathrm{BF}$ of the shell

$$
\begin{aligned}
\vec{v}_{\mathrm{BF}, \mathrm{s}}= & R_{z}\left[\chi_{s}\right] R_{x}\left[-\epsilon_{s}\right] R_{z}\left[\phi_{s}\right] R_{x}\left[\theta_{s}\right] R_{z}\left[\xi_{s}\right] R_{z}\left[-\xi_{i}\right] R_{x}\left[-\theta_{i}\right] \\
& \times R_{z}\left[-\phi_{i}\right] R_{x}\left[\epsilon_{i}\right] R_{z}\left[-\chi_{i}\right] \vec{v}_{\mathrm{BF}, \mathrm{i}} .
\end{aligned}
$$

The first two rotations $R_{z}\left[-\chi_{i}\right]$ and $R_{x}\left[\epsilon_{i}\right]$ express the vector $\vec{v}$ in the reference frame related to the rotation axis of the interior (see Fig. 3). We then express this vector in the inertial frame by applying the three rotations $R_{z}\left[-\phi_{i}\right], R_{x}\left[-\theta_{i}\right]$ and $R_{z}\left[-\xi_{i}\right]$. Finally, we use the rotations $R_{z}\left[\xi_{s}\right], R_{x}\left[\theta_{s}\right], R_{z}\left[\phi_{s}\right], R_{x}\left[-\epsilon_{s}\right]$ and $R_{z}\left[\chi_{s}\right]$ to express the vector $v$ in the $\mathrm{BF}$ of the shell, through the intermediate frame related to the rotation axis of the shell. The transition matrix $\mathbf{R}_{\mathrm{BF}}$ between the $\mathrm{BF}$ of the shell and the $\mathrm{BF}$ of the interior can then, correct up to the first order in the small quantities, be expressed as

$\mathbf{R}_{\mathrm{BF}}=\left(\begin{array}{ccc}1 & l_{2} & l_{3} \\ -l_{2} & 1 & m_{3} \\ -l_{3} & -m_{3} & 1\end{array}\right)$.

As any rotation matrix, $\mathbf{R}_{\mathrm{BF}}$ is an orthogonal matrix at the first order in the small quantities. The different components of the matrix $\mathbf{R}_{\mathrm{BF}}$ are given by

$l_{2}=\gamma_{s}-\gamma_{i}$

$l_{3}=\Delta \omega^{x} / n-\Delta \eta \sin (M+\omega)$

$m_{3}=\Delta \omega^{y} / n-\Delta \eta \cos (M+\omega)$,

where $\Delta \eta=\eta_{s}-\eta_{i}$ and $\Delta \omega^{x / y}=\omega_{x / y, s}-\omega_{x / y, i}$. Performing the volume integration Eq. (C.1), we then obtain the following expression for the gravitational torque acting on an internal layer $I$, correct up to the first order in $\Delta \eta$ and $\Delta \omega_{x / y}$

$$
\begin{aligned}
\Gamma_{g, I, X}= & -3\left(\phi_{20}+2 \phi_{22}\right)\left(C_{I}-B_{I}\right)\left[\Delta \omega^{y} / n-\Delta \eta \cos (M+\omega)\right] \\
& -3\left(\phi_{20}+2 \phi_{22}\right) c_{23}^{I}-3 \delta \phi_{21}^{\sin }\left(C_{I}-B_{I}\right)
\end{aligned}
$$

$$
\begin{aligned}
\Gamma_{g, I, y}= & 3\left(\phi_{20}-2 \phi_{22}\right)\left(C_{I}-A_{I}\right)\left[\Delta \omega^{x} / n-\Delta \eta \sin (M+\omega)\right] \\
& +3\left(\phi_{20}-2 \phi_{22}\right) c_{13}^{I}+3 \delta \phi_{21}^{\cos }\left(C_{I}-A_{I}\right)
\end{aligned}
$$

$\Gamma_{g, I, Z}=12 \phi_{22}\left(\gamma_{s}-\gamma_{i}\right)\left(B_{I}-A_{I}\right)+6 \delta \phi_{22}^{r}\left(B_{I}-A_{I}\right)+12 \phi_{22} C_{12}^{I}$.

Using the basic principle of action and reaction, the gravitational torque acting on an outer layer $O$ due to the presence of an inner layer $I$ is equal and opposite to the torque acting on the layer $I$ due to the presence of the layer $O$

$\vec{\Gamma}_{g, O}=-\vec{\Gamma}_{g, I}$.

To obtain the obliquity flattenings $\alpha \delta h_{\mathrm{obl}}^{\sin / \cos , k}$ and the librational flattening $\alpha \delta h_{k}^{l}$ of layer $k$ appearing in the gravitational term $\delta \phi_{21}^{\sin / \cos }$, we express the radial displacement $\zeta_{k}$ of layer $k$ related to the obliquity bulge of that layer as, using Eq. (C.3)

$\zeta_{k}^{\mathrm{obl}, \mathrm{cos}}=-\frac{2}{3} R_{k} \alpha \delta h_{k}^{\mathrm{obl}, \cos } R_{21}$

$\zeta_{k}^{\mathrm{obl}, \sin }=-\frac{2}{3} R_{k} \alpha \delta h_{k}^{\mathrm{obl}, \sin } S_{21}$ 
$\zeta_{k}^{l}=\frac{1}{6} R_{k} \beta \delta h_{k}^{l} S_{22}$

Using the radial tidal displacement $y_{k}$ of that layer for an unit tidal potential and the obliquity part of the tidal potential (see Coyette et al., 2016), we then find, for each layer $k$

$$
\begin{aligned}
& \alpha \delta h_{k}^{\mathrm{obl}, \cos }=-\frac{3}{2} \frac{y_{k}}{R_{k}} n^{2} R^{2}\left(\frac{4}{3} \frac{\omega_{k}^{x}}{n}-\eta_{k} \sin (M+\omega)\right) \\
& \alpha \delta h_{k}^{\mathrm{obl}, \sin }=-\frac{3}{2} \frac{y_{k}}{R_{k}} n^{2} R^{2} \frac{\omega_{k}^{y}}{3 n} \\
& \beta \delta h_{k}^{l}=6 \frac{y_{k}}{R_{k}} n^{2} R^{2}\left(e \sin (M)-\frac{\gamma_{k}}{2}\right) .
\end{aligned}
$$

\section{Appendix D. Matrices coefficients}

The coefficients appearing in the equations governing the longitudinal librations (Eqs. (41)-(43)) are given by (Van Hoolst et al., 2013)

$$
\begin{aligned}
K_{0}= & n^{2}\left(k_{2, o b}+k_{2, o t}\right) q_{r} M_{T} R^{2} \\
K_{1}= & 2\left(K_{s}+K-K_{i s}\right) \\
K_{2}= & 2\left(K_{i i}-K\right) \\
K_{3}= & K_{s}-K_{i M}+K_{s z} \\
K_{4}= & 2\left(K_{i s}-K\right) \\
K_{5}= & 2\left(K_{i}+K-K_{i i}\right) \\
K_{i i}= & \frac{4 \pi G}{5}\left(k_{2}^{i}+k_{2}^{\mathrm{ob}}\right) q_{r} M_{T} R^{2}\left[\rho_{o} \beta_{o}+\rho_{s}\left(\beta_{s}-\beta_{o}\right)\right] \\
K_{6}= & \frac{n_{i}}{4} \frac{k_{2}^{i}}{k_{f}^{i}}\left(B_{i}-A_{i}\right) \\
K_{i}= & \frac{3 n^{2}}{2}\left[\left(B_{i}-A_{i}\right) \frac{k_{f}^{i}-k_{2}^{i}}{k_{f}^{i}}+\left(B_{\mathrm{ob}}-A_{\mathrm{ob}}\right) \frac{k_{f}^{\mathrm{ob}}-k_{2}^{\mathrm{ob}}}{k_{f}^{\mathrm{ob}}}\right] \\
K_{s}= & \frac{4 \pi G}{5}\left[\left(B_{i}-A_{i}\right)+\left(B_{\mathrm{ob}}-A_{\mathrm{ob}}\right)\right]\left[\rho_{o} \beta_{o}+\rho_{s}\left(\beta_{s}-\beta_{o}\right)\right] \\
K_{f} & \left.\left(B_{s}-A_{s}\right) \frac{k_{f}^{s}-k_{2}^{s}}{k_{f}^{s}}+\left(B_{\mathrm{ot}}-A_{\mathrm{ot}}\right) \frac{k_{f}^{\mathrm{ot}}-k_{2}^{\mathrm{ot}}}{k_{f}^{\mathrm{ot}}}\right]
\end{aligned}
$$

$K_{i s}=\frac{6 \pi G}{5}\left[\left(B_{i}-A_{i}\right)+\left(B_{\mathrm{ob}}-A_{\mathrm{ob}}\right)\right] n^{2} R^{2}\left[\rho_{s}\left(\frac{y_{s}}{R}-\frac{y_{o}}{R_{o}}\right)+\rho_{o} \frac{y_{o}}{R_{o}}\right]$

$K_{i M}=K_{i s}-K_{i i}$.

The matrices $\mathbf{K}$ and $\mathbf{N}$ appearing in the equations governing the polar motion (Eq. (51)) are

$\mathbf{K}=\left(\begin{array}{cccccc}0 & K_{12} & 0 & K_{14} & 0 & K_{16} \\ K_{21} & 0 & K_{23} & 0 & K_{25} & 0 \\ 0 & K_{32} & 0 & K_{34} & 0 & 0 \\ K_{41} & 0 & K_{43} & 0 & 0 & 0 \\ 0 & K_{52} & 0 & K_{54} & 0 & K_{56} \\ K_{61} & 0 & K_{63} & 0 & K_{65} & 0\end{array}\right)$,

$\mathbf{N}=\left(\begin{array}{cccccc}N_{11} & 0 & 0 & 0 & N_{15} & 0 \\ 0 & N_{22} & 0 & 0 & 0 & N_{26} \\ N_{31} & 0 & 0 & 0 & N_{34} & 0 \\ 0 & N_{42} & 0 & 0 & 0 & N_{46} \\ N_{51} & 0 & 0 & 0 & N_{55} & 0 \\ 0 & N_{62} & 0 & 0 & 0 & N_{66}\end{array}\right)$,

where the $K_{i j}$ and $N_{i j}$ matrices coefficients are given by

$K_{12}=n\left(C_{s}-\tilde{B}_{s}\right)+\kappa_{y}-\kappa_{s, x}-\frac{1}{3} \kappa_{o t}+h_{z}(0)$

$K_{14}=n\left(C_{o t}-B_{o t}\right)$

$K_{16}=-\kappa_{y}+\kappa_{i, x}$

$K_{21}=4 n\left(\tilde{A}_{s}-C_{s}\right)+3 n\left(\tilde{A}_{o t}-C_{o t}\right)-\kappa_{x}+4 / 3\left(\kappa_{s, y}+\kappa_{o t}\right)-h_{z}(0)$

$K_{23}=n\left(A_{o t}-C_{o t}\right)$

$K_{25}=\kappa_{x}-\frac{4}{3} \kappa_{i, y}$

$K_{32}=n C_{0}$

$K_{34}=-n C_{0}$

$K_{41}=-n C_{0}$

$K_{43}=n C_{0}$

$K_{52}=-n\left(C_{o b}-B_{o b}\right)-\kappa_{y}+\kappa_{s, x}$

$K_{54}=n\left(C_{o b}-B_{o b}\right)$

$K_{56}=n\left(C_{i}-\tilde{B}_{i}+C_{o b}-\tilde{B}_{o b}\right)+\kappa_{y}-\kappa_{i, x}$

$K_{61}=n\left(C_{o b}-A_{o b}\right)+\kappa_{x}-\frac{4}{3} \kappa_{s, y}$ 
$K_{63}=n\left(A_{o b}-C_{o b}\right)$

$K_{65}=4 n\left(\tilde{A}_{i}-C_{i}+\tilde{A}_{o b}-C_{o b}\right)-\kappa_{x}+\frac{4}{3} \kappa_{i, y}$,

for the matrix $\mathbf{K}$ and

$N_{11}=-n\left(\kappa_{y}+\kappa_{s}\right)$

$N_{15}=n \kappa_{y}$

$N_{22}=3 n^{2}\left(C_{s}-\tilde{A}_{s}+C_{o t}-\tilde{A}_{o t}\right)+n\left(\kappa_{x}-\kappa_{s, y}\right)$

$N_{26}=-n\left(\kappa_{x}-\kappa_{i, y}\right)$

$N_{31}=-n^{2}\left(C_{o b}-A_{o b}\right)-n \kappa_{o t}$

$N_{35}=n^{2}\left(C_{o b}-A_{o b}\right)-n \kappa_{o b}$

$N_{42}=n^{2}\left(C_{o b}-B_{o b}\right)$

$N_{46}=-n^{2}\left(C_{o b}-B_{o b}\right)$

$N_{51}=n^{2}\left(C_{o b}-B_{o b}\right)+n \kappa_{y}$

$N_{55}=-n^{2}\left(C_{o b}-B_{o b}\right)-n\left(\kappa_{y}+\kappa_{i}\right)$

$N_{62}=-n^{2}\left(C_{o b}-A_{o b}\right)+n\left(\kappa_{s, y}-\kappa_{x}\right)$

$N_{66}=3 n^{2}\left(C_{i}-\tilde{A}_{i}\right)+4 n^{2}\left(C_{o b}-\tilde{A}_{o b}\right)+n\left(\kappa_{x}-\kappa_{i, y}\right)$,

for the matrix $\mathbf{N}$. The different $\kappa$ coefficients appearing in Eqs. (D.18)-(D.33) are given by

$\begin{aligned} \kappa_{x}= & \frac{4 \pi G}{5 n}\left(C_{i}+C_{o b}-A_{i}-A_{o b}\right)\left[\rho_{o}\left(2 \alpha_{o}+\beta_{o}\right)\right. \\ & \left.+\rho_{s}\left(2 \alpha_{s}+\beta_{s}-2 \alpha_{o}-\beta_{o}\right)\right]\end{aligned}$

$\kappa_{y}=\frac{4 \pi G}{5 n}\left(C_{i}+C_{o b}-B_{i}-B_{o b}\right)\left[\rho_{o}\left(2 \alpha_{o}-\beta_{o}\right)\right.$

$\left.+\rho_{s}\left(2 \alpha_{s}-\beta_{s}-2 \alpha_{o}+\beta_{0}\right)\right]$

$\kappa_{s, x}=\frac{4 \pi G}{5} n R^{2}\left(C_{i}+C_{o b}-B_{i}-B_{o b}\right)\left(\rho_{s} \frac{y_{s}}{R}-\left(\rho_{s}-\rho_{o}\right) \frac{y_{o}}{R_{o}}\right)$

$\kappa_{s, y}=\frac{12 \pi G}{5} n R^{2}\left(C_{i}+C_{o b}-A_{i}-A_{o b}\right)\left(\rho_{s} \frac{y_{s}}{R}-\left(\rho_{s}-\rho_{o}\right) \frac{y_{o}}{R_{o}}\right)$

$\kappa_{i, x}=\frac{4 \pi G}{15 n} q_{r} M_{T} R^{2}\left(k_{2, i}+k_{2, o b}\right)\left[\rho_{o}\left(2 \alpha_{o}-\beta_{o}\right)\right.$

$\left.+\rho_{s}\left(2 \alpha_{s}-\beta_{s}-2 \alpha_{o}+\beta_{0}\right)\right]$

$$
\begin{aligned}
\kappa_{i, y}= & \frac{4 \pi G}{5 n} q_{r} M_{T} R^{2}\left(k_{2, i}+k_{2, o b}\right)\left[\rho_{o}\left(2 \alpha_{o}+\beta_{o}\right)\right. \\
& \left.+\rho_{s}\left(2 \alpha_{s}+\beta_{s}-2 \alpha_{o}-\beta_{o}\right)\right]
\end{aligned}
$$

$\kappa_{k}=n k_{2, k} q_{r} M_{T} R^{2}$,

for $k=s, k=i, k=o t$ and $k=o b$.

\section{References}

Baland, R.-M., Tobie, G., Lefèvre, A., Van Hoolst, T., 2014. Titan's internal structure inferred from its gravity field, shape, and rotation state. Icarus 237, 29-41.

Baland, R.-M., Van Hoolst, T., Yseboodt, M., Karatekin, O., 2011. Titan's obliquity as evidence of a subsurface ocean? Astron. Astrophys. 530, A141. doi:10.1051/ 0004-6361/201116578.

Baland, R.-M., Yseboodt, M., Van Hoolst, T., 2016. The obliquity of enceladus. Icarus 268, 12-31.

Barr, A.C., Showman, A.P., 2009. Heat transfer in Europa's icy shell. In: Pappalardo, R.T., McKinnon, W.B., Khurana, K.K. (Eds.), Europa. University of Arizona Press, Tucson, p. 405.

Béghin, C., Randriamboarison, O., Hamelin, M., Karkoschka, E., Sotin, C., Whitten, R.C., Berthelier, J.J., Grard, R., Simões, F., 2012. Analytic theory of Titan's schumann resonance: constraints on ionospheric conductivity and buried water ocean. Icarus 218 (2), 1028-1042. doi:10.1016/j.icarus.2012.02.005.

Beuthe, M., 2013. Spatial patterns of tidal heating. Icarus 223, 308-329.

Beuthe, M., Rivoldini, A., Trinh, A., 2016. Enceladus's and dione's floating ice shells supported by minimum stress isostasy. Geophys. Res. Lett. 43 (19), 10.08810.096. doi:10.1002/2016GL070650. 2016GL070650.

Bills, B.G., Nimmo, F., 2008. Forced obliquity and moments of inertia of titan. Icarus 196 (1), 293-297. doi:10.1016/j.icarus.2008.03.002.

Boué, G., Rambaux, N., Richard, A., 2017. Rotation of a rigid satellite with a fluid component: a new light onto titan?s obliquity. Celest. Mech. Dyn. Astron. 129, 449-485.

Buffett, B., 1996. Gravitational oscillations in the length of day. Geophys. Res. Lett. 23 (17), 2279-2282

Choukroun, M., Sotin, C., 2012. Is Titan's shape caused by its meteorology and carbon cycle? Geophys. Res. Lett. 39 (4). doi:10.1029/2011GL050747. L04201.

Cole, D.M., Durell, G.D., 1995. The cyclic loading of saline ice. Philos. Mag. A 72 (1), 209-229, doi:10.1080/01418619508239591.

Coyette, A., Hoolst, T.V., Baland, R.-M., Tokano, T., 2016. Modeling the polar motion of titan. Icarus 265, 1-28. doi:10.1016/j.icarus.2015.10.015.

Croft, S., Lunine, J., Kargel, J., 1988. Equation of state of ammonia-water liquid: derivation and planetological applications. Icarus 73 (2), 279-293. doi:10.1016/ 0019-1035(88)90098-X.

Dehant, V., Mathews, P.M., 2015. Precession, Nutation and Wobble of the Earth. Cambridge University Press, Cambridge.

Dumberry, M., Rivoldini, A., Hoolst, T.V., Yseboodt, M., 2013. The role of Mercury's core density structure on its longitudinal librations. Icarus 225 (1), 62-74. doi:10.1016/j.icarus.2013.03.001.

Dumberry, M., Wieczorek, M.A., 2016. The forced precession of the Moon's inner core. J. Geophys. Res.: Planets 121 (7), 1264-1292. doi:10.1002/2015JE004986.

Fortes, A., Grindrod, P., Trickett, S., Voadlo, L., 2007. Ammonium sulfate on titan: possible origin and role in cryovolcanism. Icarus 188 (1), 139-153. doi:10.1016 j.icarus.2006.11.002.

Fortes, A.D., 2012. Titan's internal structure and the evolutionary consequences Planet. Space Sci. 60, 10-17.

Gao, P., Stevenson, D.J., 2013. Nonhydrostatic effects and the determination of icy satellites' moment of inertia. Icarus 226 (2), 1185-1191. doi:10.1016/j.icarus. 2013.07.034.

Goldreich, P.M., Mitchell, J.L., 2010. Elastic ice shells of synchronous moons: implications for cracks on europa and non-synchronous rotation of titan. Icarus 209 (2), 631-638. doi:10.1016/j.icarus.2010.04.013.

Grasset, O., Sotin, C., Deschamps, F., 2000. On the internal structure and dynamics of titan. Planet. Space Sci. 48 (78), 617-636. doi:10.1016/S0032-0633(00)00039-8.

Greenberg, R., Weidenschilling, S., 1984. How fast do galilean satellites spin? Icarus 58 (2), 186-196. doi:10.1016/0019-1035(84)90038-1.

Hauck, S.A., Margot, J.-L., Solomon, S.C., Phillips, R.J., Johnson, C.L., Lemoine, F.G., Mazarico, E., McCoy, T.J., Padovan, S., Peale, S.J., Perry, M.E., Smith, D.E., Zuber, M.T., 2013. The curious case of Mercury's internal structure. J. Geophys. Res.: Planets 118 (6), 1204-1220. doi:10.1002/jgre.20091.

Helgerud, M.B., Waite, W.F., Kirby, S.H., Nur, A., 2009. Elastic wave speeds and moduli in polycrystalline ice ih, si methane hydrate, and sii methane-ethane hydrate. J. Geophys. Res.: Solid Earth 114 (B2). doi:10.1029/2008JB006132. B02212.

Hemingway, D., Nimmo, F., Zebker, H., Iess, L., 2013. A rigid and weathered ice shell on titan. Nature 500, 550-552.

Iess, L., Jacobson, R.A., Ducci, M., Stevenson, D.J., Lunine, J.I., Armstrong, J.W., Asmar, S.W., Racioppa, P., Rappaport, N.J., Tortora, P., 2012. The tides of titan. Science 337, 457-459.

Iess, L., Rappaport, N.J., Jacobson, R.A., Racioppa, P., Stevenson, D.J., Tortora, P., Armstrong, J.W., Asmar, S.W., 2010. Gravity field, shape, and moment of inertia of titan. Science 327, 1367-1369. 
Jara-Orué, H.M., Vermeersen, B.L.A., 2014. The forced libration of Europa's deformable shell and its dependence on interior parameters. Icarus 229, 31-44.

Lambeck, K., 1980. The Earth's Variable Rotation: Geophysical Causes and Consequences. Cambridge University Press, Cambridge.

Lorenz, R.D., 2008. Titan's rotation reveals an internal ocean and changing zonal winds. Nature 319, 1649-1651.

Margot, J., Peale, S., Jurgens, R., Slade, M., Holin, I., 2007. Large longitude libration of mercury reveals a molten core. Science 316, 710-714.

Margot, J.-L., Peale, S.J., Solomon, S.C., Hauck, S.A., Ghigo, F.D., Jurgens, R.F., Yseboodt, M., Giorgini, J.D., Padovan, S., Campbell, D.B., 2012. Mercury's moment of inertia from spin and gravity data. J. Geophys. Res.: Planets 117 (E12). doi:10.1029/2012JE004161. E00L09.

Mathews, P.M., Buffett, B.A., Herring, T.A., Shapiro, I.I., 1991. Forced nutations of the earth: influence of inner core dynamics 1. Theory. J. Geophys. Res. 96 (B5), 8219-8242.

Meriggiola, R., Iess, L., Stiles, B., Lunine, J., Mitri, G., 2016. The rotational dynamics of titan from cassini RADAR images. Icarus 275, 183-192. doi:10.1016/j.icarus. 2016.01.019.

Moritz, H., 1990. The Figure of the Earth: Theoretical Geodesy and the Earth's Interior. Wichmann, Karlsruhe.

Nimmo, F., Bills, B., 2010. Shell thickness variations and the long-wavelength topography of titan. Icarus 208 (2), 896-904. doi:10.1016/j.icarus.2010.02.020.

Noyelles, B., Nimmo, F., 2014. New clues on the interior of Titan from its rotation state. In: Complex Planetary Systems. In: Proceedings of the International Astronomical Union, 9, pp. 17-20. doi:10.1017/S1743921314007728.

Peale, S., 1969. Generalized Cassini's laws. Astron. J. 74 (3), 483-489.

Poincaré, H., 1910. Sur la précession des corps déformables. Bull. Astron. 27, 321-356.

Richard, A., Rambaux, N., Charnay, B., 2014. Librational response of a deformed 3-layer titan perturbed by non-Keplerian orbit and atmospheric couplings. Planet. Space Sci. 93-94, 22-34.

Rivoldini, A., Van Hoolst, T., 2013. The interior structure of mercury constrained by the low-degree gravity field and the rotation of mercury. Earth Planet. Sci. Lett. 377-378, 62-72. doi:10.1016/j.epsl.2013.07.021.

Sabadini, R., Vermeersen, B., 2004. Normal Mode Theory in Viscoelasticity. Springer, Dordrecht, Netherlands, pp. 1-44.

Sohl, F., Hussmann, H., Schwentker, B., Spohn, T., Lorenz, R.D., 2003. Interior structure models and tidal love numbers of titan. J. Geophys. Res. 108, 5130.

Sotin, C., Grasset, O., Beauchesne, S., 1998. Thermodynamic Properties of High Pressure Ices: Implications for the Dynamics and Internal Structure of Large Icy Satellites. Springer, Dordrecht, Netherlands, pp. 79-96.

Stiles, B.W., Kirk, R.L., Lorenz, R.D., Hensley, S., Lee, E., Ostro, S.J., Allison, M.D., Callahan, P.S., Gim, Y., Iess, L., Perci Del Marmo, P., Hamilton, G., Johnson, W.T.K., West, R.D., the Cassini RADAR Team, 2008. Determining Titan's spin state from cassini radar images. Astron. J. 135, 1669-1680.

Stiles, B.W., Kirk, R.L., Lorenz, R.D., Hensley, S., Lee, E., Ostro, S.J., Allison, M.D., Callahan, P.S., Gim, Y., Iess, L., Perci Del Marmo, P., Hamilton, G., Johnson, W.T.K. West, R.D., the Cassini RADAR Team, 2010. Erratum: determining Titan's spin state from cassini radar images. Astron. J. 139, 311.

Szeto, A.M.K., Xu, S., 1997. Gravitational coupling in a triaxial ellipsoidal earth. J. Geophys. Res. 102 (B12), 27651-27657.
Thomas, P., Tajeddine, R., Tiscareno, M., Burns, J., Joseph, J., Loredo, T., Helfenstein, P., Porco, C., 2016. Enceladus's measured physical libration requires a global subsurface ocean. Icarus 264, 37-47. doi:10.1016/j.icarus.2015.08.037.

Tobie, G., Grasset, O., Lunine, J.I., Mocquet, A., Sotin, C., 2005. Titan's internal structure inferred from a coupled thermal-orbital model. Icarus 175 (2), 496-502. doi:10.1016/j.icarus.2004.12.007.

Tokano, T., 2013. Wind-induced equatorial bulge in venus and titan general circulation models: implication for the simulation of superrotation. Geophys. Res. Lett. 40, 4538-4543.

Tokano, T., Lorenz, R.D., 2015. Wind-driven circulation in Titan's seas. J. Geophys. Res. 120. doi:10.1002/2014JE004751.

Tokano, T., Lorenz, R.D., Van Hoolst, T., 2014. Numerical simulations of tides and oceanic angular momentum of Titan's hydrocarbon seas. Icarus 242, 188-201.

Tokano, T., Neubauer, F.M., 2005. Wind-induced seasonal angular momentum exchange at Titan's surface and its influence on Titan's length-of-day. Geophys. Res. Lett. 32 (24), 20-33. doi:10.1029/2005GL024456. L24203.

Tokano, T., Van Hoolst, T., Karatekin, O., 2011. Polar motion of titan forced by the atmosphere. J. Geophys. Res. 116, E05002.

Van Hoolst, T., 2007. 10.04 - The rotation of the terrestrial planets. In: Schubert, G. (Ed.), Treatise on Geophysics. Elsevier, Amsterdam, pp. 123-164. doi:10.1016/ B978-044452748-6.00155-3.

Van Hoolst, T., 2015. 10.04 - Rotation of the terrestrial planets. In: Schubert, G. (Ed.), Treatise on Geophysics. Elsevier, Oxford, pp. 121-151. doi:10.1016/ B978-0-444-53802-4.00168-8.

Van Hoolst, T., Baland, R.-M., Trinh, A., 2013. On the librations and tides of large icy satellites. Icarus 226, 299-315

Van Hoolst, T., Baland, R.-M., Trinh, A., 2016. The diurnal libration and interior structure of enceladus. Icarus 277, 311-318. doi:10.1016/j.icarus.2016.05.025.

Van Hoolst, T., Dehant, V., 2002. Influence of triaxiality and second-order terms in flattenings on the rotation of terrestrial planets: I. Formalism and rotational normal modes. Phys. Earth Planet. Inter. 134, 17-33.

Van Hoolst, T., Rambaux, N., Karatekin, O., Baland, R.-M., 2009. The effect of gravitational and pressure torques on titan length-of-day variations. Icarus 200, 256-264.

Van Hoolst, T., Rambaux, N., Karatekin, Ö., Dehant, V., Rivoldini, A., 2008. The librations, shape and icy shell of europa. Icarus 195, 386-399.

Vance, S., Brown, J.M., 2013. Thermodynamic properties of aqueous $\mathrm{mgso}_{4}$ to $800 \mathrm{MPa}$ at temperatures from -20 to $100 \mathrm{c}$ and concentrations to $2.5 \mathrm{~mol} \mathrm{~kg}^{-1}$ from sound speeds, with applications to icy world oceans. Geochim. Cosmochim. Acta 110, 176-189. doi:10.1016/j.gca.2013.01.040.

Čadek, O., Tobie, G., Van Hoolst, T., Massé, M., Choblet, G., Lefèvre, A., Mitri, G. Baland, R.-M., Běhounková, M., Bourgeois, O., Trinh, A., 2016. Enceladus'S internal ocean and ice shell constrained from cassini gravity, shape, and libration data. Geophys. Res. Lett. 43 (11), 5653-5660. doi:10.1002/2016GL068634. 2016 GL068634.

Yseboodt, M., Van Hoolst, T., 2014. The long-period forced librations of Titan. In: Complex Planetary Systems. In: Proceedings of the International Astronomical Union, 9, pp. 25-28.

Zebker, H.A. Stiles, B., Hensley, S., Lorenz, R., Kirk, R.L., Lunine, J., 2009. Size and shape of Saturn's moon titan. Science 324, 921-923. 